\title{
Methylmercury displays pro-adipogenic properties in rainbow trout preadipocytes
}

\author{
Gilles Tinant ${ }^{\mathrm{a},{ }^{*}, \text { Ineke Neefs }}{ }^{\mathrm{a}}$, Krishna Das ${ }^{\mathrm{b}}$, Jean-François Rees ${ }^{\mathrm{a}}$, Yvan Larondelle ${ }^{\mathrm{a}}$, \\ Cathy Debier ${ }^{\text {a, ** }}$ \\ ${ }^{a}$ Louvain Institute of Biomolecular Science and Technology (LIBST), Université catholique de Louvain, Croix Du Sud 4-5/L7.07.03, 1348, Louvain-la-Neuve, \\ Belgium \\ ${ }^{\mathrm{b}}$ Laboratory of Oceanology, Université de Liège, 11 Allée Du 6 Août, B6C, 4000, Liège, Belgium
}

\section{H I G H L I G H T S}

- MeHg induced a dose-dependent accumulation of neutral lipids in preadipocytes.

- Accumulation of lipids persisted beyond the cellular exposure to MeHg.

- MeHg increased n-3 PUFA levels in lipid droplets and dropped AA levels in membranes.

- Expression of adipocyte- and lipid-specific genes was modulated by MeHg.

- Total mercury accumulated in cells was released when MeHg exposure stopped.

\section{A R T I C L E I N F O}

\section{Article history:}

Received 23 May 2020

Received in revised form

27 July 2020

Accepted 3 August 2020

Available online 17 August 2020

Handling Editor: Jim Lazorchak

\section{Keywords:}

Preadipocyte

Methylmercury

Adipogenesis

Fatty acid

\begin{abstract}
A B S T R A C T
Methylmercury ( $\mathrm{MeHg}$ ) is a ubiquitous contaminant largely found in aquatic environments, especially in species at high trophic level such as salmonids. The aim of this study was to evaluate the effects of MeHg on adipocyte differentiation and lipid metabolism in rainbow trout. Primary cultured preadipocytes were exposed to increasing concentrations of $\mathrm{MeHg}$ during six days with or without a hormonal cocktail. Main results showed a dose-dependent intracellular accumulation of neutral lipids with a preferential uptake of $n-3$ polyunsaturated fatty acids. Interestingly, this accumulation occurred after a fairly low uptake of $\mathrm{MeHg}$ by preadipocytes and was maintained after the cellular exposure to MeHg. In membrane phospholipids, arachidonic acid (20:4 n-6) was released in a dose-dependent manner. At the transcriptional level, the expression of several adipocyte-specific genes (perilipin 2 and apolipoprotein $\mathrm{Eb}$ ) as well as lipid-related genes (fatty acid synthase and fatty acid binding protein 11a) was up-regulated in preadipocytes exposed to $\mathrm{MeHg}$. These results highlight for the first time the disrupting effect of $\mathrm{MeHg}$ in trout adipocyte metabolism, providing new insights regarding the role of environmental pollutants in adipose tissue dysfunction and related pathologies.
\end{abstract}

(C) 2020 Elsevier Ltd. All rights reserved.

Abbreviations: AA, arachidonic acid (20:4 n-6); ACLY, adenosine triphosphate citrate lyase; ACSL1, long-chain acyl-CoA synthetase 1; ApoEb, apolipoprotein Eb; C/EBP $\alpha$

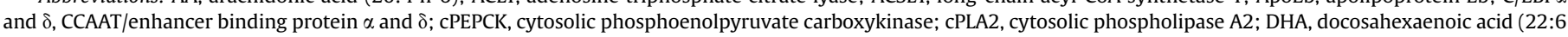

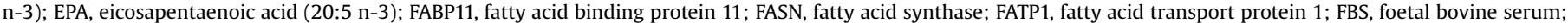

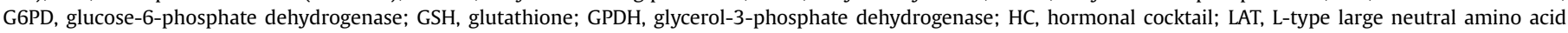

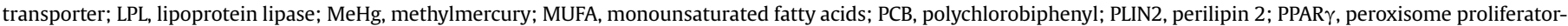
activated receptor $\gamma$; PUFA, polyunsaturated fatty acids; ROS, reactive oxygen species; SFA, saturated fatty acids; TBT, tributyltin; THg, total mercury; TPT, triphenyltin.

* Corresponding author.

** Corresponding author.

E-mail addresses: gilles.tinant@uclouvain.be (G. Tinant), cathy.debier@uclouvain.be (C. Debier). 


\section{Introduction}

Obesity has become a pandemic affecting adults and children in developed and in developing countries (WHO, 2018). This multifactorial disease is characterized by an abnormal or excessive fat accumulation. Its development is influenced by various factors such as nutrition, physical activity, genetics, microbiota and environment (reviewed by Heindel and Blumberg (2018)). Adipose tissue represents the major site of energy storage in the body and it is therefore a key organ in obesity development. This tissue is mainly made up of adipocytes with a lipid droplet filled with triglycerides. It has important roles in energy homeostasis and endocrine regulation (Harwood, 2012). The contribution of environmental chemicals to the development of obesity was highlighted since the last decade. In this context, the term "obesogen" was defined by Grün and Blumberg in 2006 as chemicals that inappropriately alter lipid homeostasis to promote adipogenesis and lipid accumulation (reviewed by Grün and Blumberg (2009)).

Adipogenesis is a tightly regulated process including the proliferation of preadipocytes followed by their differentiation into mature adipocytes. Numerous transcription factors are involved in the differentiation of preadipocytes. Peroxisome proliferatoractivated receptor $\gamma$ (PPAR $\gamma$ ) is the key one. After induction of its expression by CCAAT/enhancer binding protein $\beta$ and $\delta$ (C/EBP $\beta$ and $\delta)$, PPAR $\gamma$ induces the expression of $C / E B P \alpha$, which exerts a positive feedback on the expression of PPAR $\gamma$ (Rosen et al., 2000). This transcriptional cascade activates the expression of adipocyterelated genes and leads to the differentiated adipocyte phenotype. Various compounds such as polychlorobiphenyl (PCB) and polybrominated diphenyl ether congeners, bisphenol A or tributyltin (TBT) have been reported as obesogens in in vitro and in vivo animal and human models (Newbold et al., 2009; Ferrante et al., 2014; Ariemma et al., 2016; Lutfi et al., 2017). Obesogens mainly act through increasing the number or the size of adipocytes, or altering the endocrine regulation and the lipid homeostasis (Darbre, 2017).

Methylmercury ( $\mathrm{MeHg}$ ) is a toxic and widely distributed organo-metal (Bjørklund et al., 2017). MeHg is a well-known neurotoxicant in mammals and fish but can also affect liver and kidney notably through the generation of oxidative stress (Farina et al., 2011; Kidd and Batchelar, 2011). Humans are mainly exposed to $\mathrm{MeHg}$ through the consumption of contaminated seafood. Indeed, $\mathrm{MeHg}$ can bioaccumulate throughout the aquatic food chain (Lavoie et al., 2013) and high levels of contamination can be found in aquatic species, like salmonids (Schwindt et al., 2008). However, little is known about the potential impacts of $\mathrm{MeHg}$ on adipose tissue. To the best of our knowledge, only three studies have investigated this problematic. It has been shown that MeHg caused an alteration of structure and endocrine function in 3T3-L1 cell line (Vertigan et al., 2015; Chauhan et al., 2019). On the other hand, $\mathrm{MeHg}$ did not affect lipid accumulation in human preadipocytes and mesenchymal stem cells up to $100 \mathrm{nM}$ (van den Dungen et al., 2017). If the impact of $\mathrm{MeHg}$ has only been scarcely investigated, several studies highlighted the impairment of adipogenesis by inorganic mercury via in vitro and in vivo experiments in rodents (Barnes et al., 2003; Kawakami et al., 2012; Rizzetti et al., 2019). MeHg has also been shown to affect cellular energy metabolism in other organs. Transcriptomic and proteomic analyses of $\mathrm{MeHg}$ treated fish liver revealed modulation of lipid metabolism and particularly fatty acid biosynthesis and $\beta$-oxidation pathways (Klaper et al., 2008; Yadetie et al., 2016).

In the present study, the impacts of $\mathrm{MeHg}$ were investigated using an original in vitro model of rainbow trout preadipocytes. This species is a well-known model to study the impact of pollution (Schirmer, 2006; Ríos et al., 2015). In addition, fish adipose tissue metabolism is close to the one of humans, among others because it also uses circulating fatty acid to synthesize triglycerides, contrary to rodents. This study aims to provide for the first time a comprehensive set of data encompassing both the accumulation and release of $\mathrm{MeHg}$ in/from the adipose tissue as well as the effects of this toxic compound on the development and lipid metabolism of rainbow trout preadipocytes.

\section{Material \& methods}

\subsection{Experimental design}

An in vitro model of primary culture of rainbow trout (Oncorhynchus mykiss) preadipocytes was developed to investigate the impacts of $\mathrm{MeHg}$ on preadipocytes. Two consecutive experiments were conducted. In Experiment 1, preadipocytes were exposed to $\operatorname{MeHg}(0,0.3,1.7$ and $3.8 \mu \mathrm{M})$ with or without a hormonal cocktail (HC) during six days. The total mercury (THg) load and the fatty acid composition of the cells (phospholipids and neutral lipids) were assessed at the end of the exposure. The expression of several genes involved in adipogenesis and lipogenesis was measured after two and six days of $\mathrm{MeHg}$ exposure. As the most significant effects were observed at the highest $\mathrm{MeHg}$ concentration and with the HC, Experiment 2 was carried out with these conditions. The objective of the second experiment was to evaluate the effect of exposure duration on mercury and lipid accumulation and to investigate the potential efflux of MeHg from the preadipocytes. Confluent cells were exposed to $\mathrm{MeHg}$ and HC during two, four or six days. Cells from the first two exposure time conditions (i.e. two and four days) were then maintained in a non-contaminated medium until day 6 .

\subsection{Animals and ethics statement}

The experiments were approved by the Animal Care and Use Committee of the Université catholique de Louvain (Permit number: 153201). Female rainbow trout eggs were obtained from 'Pisciculture Charles Murgat' (Beaufort, France) and were raised in flow-through system at the 'Plateforme technologique en biologie aquicole Marcel Huet' (Université catholique de Louvain, Louvainla-Neuve, Belgium) (Permit number for animal facilities: 1220034). Fish were maintained at $12 \pm 1{ }^{\circ} \mathrm{C}$ under natural photoperiod and fed daily with a commercial diet (Skretting, Stavanger, Norway).

\subsection{Primary culture of preadipocytes, cell differentiation and $\mathrm{MeHg}$ exposure}

The primary culture of trout preadipocytes was developed based on Bouraoui et al. (2008) and is described in detail in Appendix A. Briefly, perivisceral adipose tissue was collected from euthanized fish and digested with 400 units/mL type II collagenase (Life Technologies, Thermo Fisher Scientific, Waltham, MA, USA) and 1\% bovine serum albumin (BSA) (w:v) (Sigma-Aldrich, Saint-Louis, MO, USA). After filtration and centrifugation, cells were seeded in 6-well plates and cultured until confluence was reached (day 0 ). In Experiment 1, cells were then exposed during six days to growth medium deprived of GlutaMAX ${ }^{\mathrm{TM}}$ and supplemented with $0,0.3,1.7$ or $3.8 \mu \mathrm{M} \mathrm{MeHg}\left(\mathrm{CH}_{3} \mathrm{HgCl}\right.$; CAS 115-09-3) (Sigma-Aldrich), $4 \mu \mathrm{L} / \mathrm{mL}$ lipid mixture (Sigma-Aldrich), and with or without a HC containing 
$10 \mu \mathrm{g} / \mathrm{mL}$ insulin (Sigma-Aldrich), $10 \mu \mathrm{M}$ ciglitizone (SigmaAldrich) and $0.25 \mu \mathrm{M}$ dexamethasone (Sigma-Aldrich). The $\mathrm{MeHg}$ concentrations were chosen within a range of environmentally relevant concentrations encountered in fish and marine mammals (Wagemann et al., 1998; Mahaffey et al., 2004). In Experiment 2, cells were then exposed during two, four or six days to growth medium deprived of GlutaMAX ${ }^{\mathrm{TM}}$ and supplemented with $3.8 \mu \mathrm{M}$ $\mathrm{MeHg}, 4 \mu \mathrm{L} / \mathrm{mL}$ lipid mixture and HC. After two and four days of exposure, cells were submitted to the same medium but deprived in MeHg until day 6. Lipid mixture was composed of $4.5 \mathrm{~g} / \mathrm{L}$ cholesterol, $10 \mathrm{~g} / \mathrm{L}$ cod liver oil fatty acid methyl esters, $25 \mathrm{~g} / \mathrm{L}$ polyoxyethylenesorbitan monooleate, and $2 \mathrm{~g} / \mathrm{L}$ D- $\alpha$-tocopherol acetate. Medium was renewed every $48 \mathrm{~h}$ during the 6-day exposure period. Three independent cultures were carried out with seven fish for each of them. The cytotoxicity of contaminated medium was assessed after two, four and six days of exposure by measuring the release of lactate dehydrogenase ( $\mathrm{LDH}$ ) into the extracellular medium. The activity of LDH was quantified with the Cytotoxicity Detection Kit from Roche Diagnostics (Basel, Switzerland). No cytotoxicity was observed at any time and any $\mathrm{MeHg}$ concentration (maximum 6.5\% of additional released LDH compared to negative control) (data not shown).

\subsection{THg quantification}

THg was quantified in cells and in culture media by atomic absorption spectrophotometry with Direct Mercury Analyzer 80 (DMA-80) (Milestone, Sorisole, Italy) (Habran et al., 2013). After medium removal and cell washing with phosphate-buffered saline (PBS) at $19{ }^{\circ} \mathrm{C}$, cells were harvested in $300 \mu \mathrm{L}$ of lysis buffer composed of $35 \mathrm{mM}$ sodium dodecyl sulfate, $60 \mathrm{mM}$ Tris buffer and $10 \mathrm{mM}$ sodium EDTA. Media were processed with DMA-80 without any additional treatment. All samples were stored in glass containers. Blanks and $\mathrm{Hg}$ standards (in range of the calibration curve) were included in analysis. Repeatability of measurements was verified with technical duplicates.

\subsection{Determination of fatty acid composition and concentrations}

One hundred $\mu \mathrm{L}$ of cell lysate were sampled for fatty acid quantification. Total lipids were first extracted with methanol:chloroform:water (2:2:1.8, v:v:v) (Bligh and Dyer, 1959). An internal standard composed of 1,2-dipentadecanoyl-sn-glycero-3phosphatidylcholine (Larodan, Solna, Sweden) and triheptadecanoin (Larodan) was added in each sample. Extracted lipids were then loaded on solid phase extraction columns (Bond Elut- $\mathrm{NH}_{2}$, $200 \mathrm{mg}, 3 \mathrm{~mL}$ ) (Agilent Technologies, Santa Clara, CA, USA) and neutral lipid and phospholipid fractions were eluted with chlorofom:2-propanol (2:1; v:v) and methanol, respectively (Schneider et al., 2012). Each fraction was then methylated according to Schneider et al. (2012) with minor modifications. Briefly, samples were methylated in $\mathrm{KOH}$ solution in methanol $(0.1 \mathrm{M})$ at $70{ }^{\circ} \mathrm{C}$ for $1 \mathrm{~h}$ and then in $\mathrm{HCl}$ solution in methanol $(1.2 \mathrm{M})$ at $70{ }^{\circ} \mathrm{C}$ for $15 \mathrm{~min}$. Fatty acid methyl esters (FAMEs) were extracted with hexane and methyl-undecanoate (Larodan) was added in each sample as injection standard. FAMEs were analysed by gas chromatography (Trace 1310) (Thermo Fisher Scientific, Milan, Italy). All technical details are provided in Appendix A. Results are expressed in $\mu \mathrm{mol}$ of fatty acids per $g$ of cellular proteins. Proteins were quantified in the same samples than fatty acids with Pierce ${ }^{\mathrm{TM}}$ Bicinchoninic acid Protein Assay Kit (Thermo Fisher Scientific, USA). A standard curve of BSA was used for quantification. Besides cellular samples, fatty acid composition of foetal bovine serum (FBS) and lipid mixture was also determined but without separation in lipid fractions. The percentage of increase or decrease of fatty acids due to treatments was calculated as illustrated in Mauderly and Samet (2008).

\subsection{RNA extraction, cDNA synthesis and RT-PCR analysis}

Cells were collected following the recommendations of the manufacturer in one $\mathrm{mL}$ TRIzol ${ }^{\circledR}$ Reagent (Life Technologies) per well. RNA was then extracted with Aurum ${ }^{\mathrm{TM}}$ Total RNA Mini Kit from Bio-Rad Laboratories (Hercules, CA, USA) and reverse transcribed into cDNA with the iScript ${ }^{\mathrm{TM}}$ cDNA Synthesis Kit (Bio-Rad Laboratories) as detailed in the Appendix A. Real-time reverse transcription polymerase chain reaction (RT-PCR) analysis was performed with a StepOnePlus ${ }^{\mathrm{TM}}$ Real-Time PCR System (Life Technologies) in technical duplicates. Sample preparation and RTPCR analysis are described in detail in Appendix A. Amplification results were processed by $2^{-\Delta \Delta C t}$ method (Livak and Schmittgen, 2001). Owing to its high stability across treatments, $18 \mathrm{~S}$ gene was used as reference gene. Control cells (i.e. exposed to neither $\mathrm{MeHg}$ nor $\mathrm{HC}$ ) at day 2 were used as reference condition. The primers features are summarized in Table A.1.

\subsection{Statistical analyses}

Statistical analyses were performed using $\mathrm{R}$ version 3.5.1 and JMP ${ }$ Pro 14.0 softwares. The lme function from the nlme package was used in $\mathrm{R}$ to estimate a linear mixed model for each parameter (Pinheiro et al., 2018) and to take into account the relation between the samples coming from the same primary culture. Homoscedasticity and normality of residuals were checked for all parameters. If necessary, data were transformed ( $\log 10$, inverse or root square transformations) before analysis. In case of heteroscedasticity, a correction was added to the model. Statistical tests were carried out with the contrast function in $\mathrm{R}$ (Kuhn et al., 2016) or matched pairs and Student's t-tests in JMP ${ }$. For all statistical tests, a BonferroniHolm correction was applied to take into account the multiplicity of tests via the p.adjust function in R. Results were considered significant if corrected p-value was below 0.05 .

\section{Results \& discussion}

\subsection{Model of primary cultured preadipocytes from rainbow trout}

In the present study, primary culture of preadipocytes was used to investigate for the first time, to the best of our knowledge, the impacts of $\mathrm{MeHg}$ in rainbow trout preadipocytes. This in vitro model is a relevant tool to assess the effects of potential obesogenic compounds as suggested by Lutfi et al. (2017). Specific culture conditions were applied to enable the differentiation of precursor cells. First, fatty acids were supplied to the culture, as they are essential in the induction of adipocyte differentiation in fish (Vegusdal et al., 2003; Bouraoui et al., 2008). The lipid mixture was the main source of fatty acids, whereas FBS lipids contributed to a lesser extent to the supply (see Table A.2 for fatty acid composition). Second, a HC containing insulin, ciglitizone and dexamethasone was added to half of the conditions. Previous in vitro studies demonstrated the capacity of these hormones to induce the differentiation of preadipocytes in rodents (Bourez et al., 2012b; Louis et al., 2014) and in fish (in combination with fatty acids) (Bouraoui et al., 2008; Bou et al., 2017).

In this study, the addition of $\mathrm{HC}$ induced a significant increase of cellular neutral lipid content after a 6 -day differentiation $(+142 \%$ compared to cells cultured in absence of HC) (Table 1-B). A lowered content of cellular proteins per well of culture $(-18 \%)$ was also observed in these conditions (data not shown). Moreover, the expression of adipocyte-related genes was substantially increased by the $\mathrm{HC}$ such as the gene C/EBP $\delta$, a key early regulator in 
adipogenesis (Rosen et al., 2000), after two and six days (Fig. 2). The expression of adenosine triphosphate citrate lyase (ACLY) and longchain acyl-CoA synthetase 1 (ACSL1), two enzymes involved in acyl-CoA synthesis (Fig. 1), was significantly up-regulated at day 6 in presence of HC (Fig. 2). Moreover, the expression of fatty acid binding protein $11 \mathrm{a}$ (FABP11a) and apolipoprotein $\mathrm{Eb}$ (ApoEb) was significantly higher at day 6 than at day 2 in both hormonal conditions (Fig. 2), indicating that the lipid mixture alone modulates the expression of several genes in preadipocytes. These results reflect the commitment of preadipocytes into the adipocyte lineage and the specialization into lipid storage (Bouraoui et al., 2008; Lefterova and Lazar, 2009; Salmerón et al., 2013; Li and Liu, 2014; Bou et al., 2017).

\subsection{Preadipocytes exposed to increasing concentrations of $\mathrm{MeHg}$ with or without HC (Experiment 1)}

\subsubsection{MeHg exposure and accumulation in preadipocytes}

After six days of exposure to increasing concentrations of $\mathrm{MeHg}$, preadipocytes significantly accumulated $\mathrm{MeHg}$ in a dosedependent manner (Table $1-\mathrm{A}$ ). The presence of $\mathrm{HC}$ had no significant effect on the intracellular THg level (Table $1-\mathrm{A}$ ). The dosedependent accumulation observed herein is in line with the results obtained in rainbow trout liver cells (RTL-W1 cell line, MeHg, 0.15 and $0.5 \mu \mathrm{M}, 24 \mathrm{~h}$ ) (Ferain et al., 2018). In in vivo experiments, dosedependent bioaccumulation was also observed in the entire organism of rainbow trout and zebrafish (MeHg, 0.5, 5 and $50 \mu \mathrm{g} / \mathrm{g}, 42$

Table 1

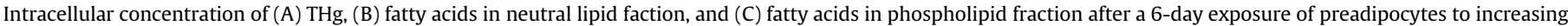

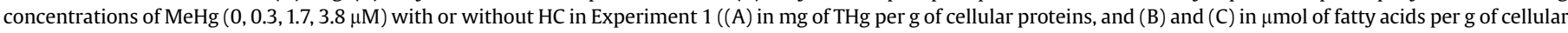
proteins).

\begin{tabular}{|c|c|c|c|c|c|c|c|c|c|}
\hline & & \multicolumn{2}{|l|}{$0 \mu \mathrm{M} \mathrm{MeHg}$} & \multicolumn{2}{|l|}{$0.3 \mu \mathrm{M} \mathrm{MeHg}$} & \multicolumn{2}{|l|}{$1.7 \mu \mathrm{M} \mathrm{MeHg}$} & \multicolumn{2}{|l|}{$3.8 \mu \mathrm{M} \mathrm{MeHg}$} \\
\hline & & Without HC & With HC & Without HC & With HC & Without HC & With HC & Without HC & With HC \\
\hline (A) & $\mathrm{THg}$ & $0.00 \pm 0.00^{\mathrm{a}}$ & $0.00 \pm 0.00^{\mathrm{A}}$ & $0.15 \pm 0.01^{b}$ & $0.15 \pm 0.02^{\mathrm{B}}$ & $1.13 \pm 0.02^{c}$ & $1.05 \pm 0.08^{C}$ & $1.84 \pm 0.07^{\mathrm{d}}$ & $1.74 \pm 0.06^{\mathrm{D}}$ \\
\hline \multirow[t]{26}{*}{ (B) } & $14: 0$ & $6.7 \pm 1.1^{\mathrm{a}}$ & $18.4 \pm 3.1 *^{\mathrm{A}}$ & $7.9 \pm 1.5^{\mathrm{a}}$ & $13.2 \pm 3.5^{\mathrm{A}}$ & $13.1 \pm 2.5^{\mathrm{a}, \mathrm{b}}$ & $25.3 \pm 7.3^{A}$ & $30.0 \pm 5.9^{b}$ & $66.8 \pm 6.5^{* B}$ \\
\hline & $16: 0$ & $31.2 \pm 2.6^{\mathrm{a}}$ & $66.5 \pm 8.3 * \mathrm{~A}$ & $34.2 \pm 6.1^{\mathrm{a}}$ & $50.8 \pm 8.4^{* \mathrm{~B}}$ & $43.3 \pm 5.7^{\mathrm{a}}$ & $83.2 \pm 20.6^{\mathrm{A}, \mathrm{B}}$ & $85.7 \pm 12.1^{\mathrm{b}}$ & $166.4 \pm 14.3 * \mathrm{C}$ \\
\hline & 18:0 & $20.5 \pm 1.4^{\mathrm{a}}$ & $29.2 \pm 2.1 * \mathrm{~A}$ & $17.4 \pm 2.4^{\mathrm{a}}$ & $21.8 \pm 1.4^{* \mathrm{~B}}$ & $18.5 \pm 2.0^{\mathrm{a}}$ & $27.8 \pm 4.3^{* \mathrm{~A}, \mathrm{~B}}$ & $28.2 \pm 2.9^{\mathrm{b}}$ & $43.4 \pm 2.3 * \mathrm{C}$ \\
\hline & Total SFA & $58.4 \pm 4.5^{a}$ & $114.1 \pm 13.4 * A$ & $59.5 \pm 9.8^{a}$ & $85.9 \pm 13.3 * B$ & $74.8 \pm 10.2^{a}$ & $136.3 \pm 32.1^{A, B}$ & $144.0 \pm 20.6^{b}$ & $276.7 \pm 23.0 * c$ \\
\hline & $16: 1 n-7$ & $6.3 \pm 1.3^{\mathrm{a}}$ & $18.6 \pm 2.9 * \mathrm{~A}, \mathrm{~B}$ & $8.7 \pm 1.8^{\mathrm{a}}$ & $13.9 \pm 4.1^{\mathrm{A}}$ & $16.3 \pm 2.6^{\mathrm{a}}$ & $29.6 \pm 8.4^{\mathrm{B}}$ & $36.1 \pm 5.5^{b}$ & $76.8 \pm 5.7 * \mathrm{C}$ \\
\hline & $18: 1 \mathrm{n}-7$ & $0.0 \pm 0.0^{\mathrm{a}}$ & $8.0 \pm 0.6^{* A}$ & $3.9 \pm 0.6^{\mathrm{b}}$ & $6.4 \pm 1.5^{\mathrm{A}}$ & $6.2 \pm 0.7^{\mathrm{a}, \mathrm{b}}$ & $12.1 \pm 3.2^{\mathrm{A}}$ & $13.3 \pm 1.5^{c}$ & $27.8 \pm 1.7 *^{* \mathrm{~B}}$ \\
\hline & $18: 1 \mathrm{n}-9$ & $34.9 \pm 5.0^{\mathrm{a}}$ & $87.9 \pm 8.2^{* A}$ & $44.1 \pm 7.6^{\mathrm{a}}$ & $72.1 \pm 17.5^{\mathrm{A}}$ & $74.1 \pm 10.5^{\mathrm{a}, \mathrm{b}}$ & $137.7 \pm 36.6^{A}$ & $152.9 \pm 22.7^{b}$ & $281.7 \pm 22.4^{* \mathrm{~B}}$ \\
\hline & $20: 1$ n-9 & $0.0 \pm 0.0^{\mathrm{a}}$ & $2.7 \pm 0.1 * \mathrm{~A}$ & $0.0 \pm 0.0^{\mathrm{a}}$ & $2.2 \pm 0.3 * \mathrm{~A}$ & $1.9 \pm 0.2^{b}$ & $3.4 \pm 0.8^{* A}$ & $3.6 \pm 0.6^{c}$ & $6.1 \pm 0.5^{* \mathrm{~B}}$ \\
\hline & $22: 1 \mathrm{n}-9$ & $6.3 \pm 1.8$ & $7.0 \pm 3.0$ & $5.4 \pm 1.6$ & $6.1 \pm 1.7$ & $5.2 \pm 1.7$ & $6.7 \pm 2.1$ & $5.8 \pm 2.4$ & $7.8 \pm 1.5$ \\
\hline & Total MUFA & $47.5 \pm 7.9^{a}$ & $124.2 \pm 14.7 * A$ & $62.0 \pm 11.4^{a}$ & $100.7 \pm 24.9 * A$ & $103.8 \pm 15.7^{a}$ & $189.5 \pm 50.4^{A}$ & $211.8 \pm 32.6^{b}$ & $400.2 \pm 31.7 *^{B}$ \\
\hline & $18: 3 n-3$ & $0.0 \pm 0.0^{\mathrm{a}}$ & $0.0 \pm 0.0^{\mathrm{A}}$ & $0.0 \pm 0.0^{\mathrm{a}}$ & $0.0 \pm 0.0^{\mathrm{A}}$ & $0.0 \pm 0.0^{\mathrm{a}}$ & $2.8 \pm 0.7 *^{* B}$ & $2.7 \pm 0.4^{b}$ & $6.8 \pm 0.4^{* C}$ \\
\hline & $18: 4 n-3$ & $0.0 \pm 0.0^{\mathrm{a}}$ & $2.6 \pm 0.4^{* A}$ & $0.0 \pm 0.0^{\mathrm{a}}$ & $1.9 \pm 0.7 * \mathrm{~A}$ & $2.0 \pm 0.3^{\mathrm{a}, \mathrm{b}}$ & $4.1 \pm 1.3^{\mathrm{A}}$ & $4.4 \pm 0.7^{b}$ & $11.2 \pm 0.8^{* \mathrm{~B}}$ \\
\hline & $20: 4 n-3$ & $0.0 \pm 0.0^{\mathrm{a}}$ & $4.0 \pm 0.5^{* A, B}$ & $2.0 \pm 0.6^{\mathrm{a}}$ & $3.1 \pm 1.0^{\mathrm{A}}$ & $3.2 \pm 0.5^{\mathrm{a}, \mathrm{b}}$ & $7.8 \pm 2.5^{\mathrm{B}}$ & $7.5 \pm 1.3^{\mathrm{b}}$ & $17.4 \pm 2.2 * \mathrm{C}$ \\
\hline & $20: 5 n-3$ & $7.9 \pm 1.8^{\mathrm{a}}$ & $21.8 \pm 2.6^{* A}$ & $11.6 \pm 2.4^{\mathrm{a}}$ & $18.1 \pm 5.4^{\mathrm{A}}$ & $21.6 \pm 3.3^{a, b}$ & $41.6 \pm 12.8^{\mathrm{A}}$ & $49.6 \pm 7.4^{\mathrm{b}}$ & $114.8 \pm 6.7 *^{* B}$ \\
\hline & $22: 5 n-3$ & $5.1 \pm 1.0^{\mathrm{a}}$ & $14.1 \pm 1.2^{\mathrm{A}}$ & $7.1 \pm 1.5^{\mathrm{a}}$ & $11.2 \pm 3.1^{\mathrm{A}}$ & $13.9 \pm 2.5^{\mathrm{a}}$ & $24.8 \pm 7.0^{* \mathrm{~B}}$ & $30.2 \pm 4.8^{\mathrm{b}}$ & $53.4 \pm 4.3 * \mathrm{C}$ \\
\hline & $24: 5 n-3$ & $0.0 \pm 0.0^{\mathrm{a}}$ & $0.0 \pm 0.0^{\mathrm{A}}$ & $0.0 \pm 0.0^{\mathrm{a}}$ & $0.0 \pm 0.0^{\mathrm{A}}$ & $0.0 \pm 0.0^{\mathrm{a}}$ & $0.0 \pm 0.0^{\mathrm{A}}$ & $1.2 \pm 0.2^{\mathrm{b}}$ & $2.1 \pm 0.1 *^{B}$ \\
\hline & $22: 6 n-3$ & $6.1 \pm 0.8^{\mathrm{a}}$ & $12.6 \pm 0.4 *^{\mathrm{A}}$ & $8.2 \pm 1.1^{\mathrm{a}}$ & $11.0 \pm 2.3^{A}$ & $15.3 \pm 2.1^{\mathrm{a}, \mathrm{b}}$ & $26.2 \pm 7.7^{\mathrm{A}}$ & $34.8 \pm 4.9^{\mathrm{b}}$ & $67.8 \pm 3.8^{* \mathrm{~B}}$ \\
\hline & Total n-3 PUFA & $19.1 \pm 3.5^{a}$ & $55.1 \pm 4.7 * A$ & $29.0 \pm 5.5^{a}$ & $45.3 \pm 12.5^{A}$ & $56.1 \pm 8.5^{a, b}$ & $107.4 \pm 31.8^{A}$ & $130.4 \pm 19.6^{b}$ & $273.6 \pm 18.1 *^{B}$ \\
\hline & $18: 2 n-6$ & $4.5 \pm 0.3^{a}$ & $11.8 \pm 0.2 * \mathrm{~A}$ & $4.9 \pm 0.4^{\mathrm{a}}$ & $8.6 \pm 1.1^{\mathrm{B}}$ & $7.5 \pm 0.4^{\mathrm{a}, \mathrm{b}}$ & $14.4 \pm 3.4^{\mathrm{A}, \mathrm{B}}$ & $14.9 \pm 2.0^{\mathrm{b}}$ & $32.2 \pm 1.4 * \mathrm{C}$ \\
\hline & $20: 3 n-6$ & $0.0 \pm 0.0^{\mathrm{a}}$ & $6.2 \pm 0.4 * \mathrm{~A}, \mathrm{~B}$ & $2.5 \pm 0.5^{\mathrm{b}}$ & $4.6 \pm 1.1 *^{\mathrm{A}}$ & $4.3 \pm 0.7^{b}$ & $8.7 \pm 2.2^{\mathrm{B}}$ & $8.6 \pm 1.3^{c}$ & $16.0 \pm 1.4^{* \mathrm{C}}$ \\
\hline & $20: 4 n-6$ & $3.1 \pm 0.2^{\mathrm{a}}$ & $9.6 \pm 0.4 *^{\mathrm{A}}$ & $4.1 \pm 0.5^{\mathrm{a}}$ & $7.3 \pm 1.6 * \mathrm{~A}$ & $6.7 \pm 0.9^{\mathrm{a}, \mathrm{b}}$ & $13.9 \pm 3.7^{\mathrm{A}}$ & $13.6 \pm 2.1^{\mathrm{b}}$ & $27.7 \pm 1.7 *^{\mathrm{B}}$ \\
\hline & $22: 4 n-6$ & $0.0 \pm 0.0^{\mathrm{a}}$ & $0.0 \pm 0.0^{\mathrm{A}}$ & $0.0 \pm 0.0^{\mathrm{a}}$ & $0.0 \pm 0.0^{\mathrm{A}}$ & $0.0 \pm 0.0^{\mathrm{a}}$ & $0.0 \pm 0.0^{\mathrm{A}}$ & $2.0 \pm 0.2^{\mathrm{b}}$ & $3.5 \pm 0.2 *^{\mathrm{B}}$ \\
\hline & Total n-6 PUFA & $7.6 \pm 0.5^{a}$ & $27.6 \pm 1 * A$ & $11.5 \pm 1.4^{a}$ & $20.5 \pm 3.9^{A}$ & $18.5 \pm 1.9^{a, b}$ & $37.0 \pm 9.3^{A}$ & $39.2 \pm 5.5^{b}$ & $79.4 \pm 4.6 * B$ \\
\hline & Total PUFA & $26.6 \pm 3.9^{a}$ & $82.7 \pm 5.5 * A$ & $40.5 \pm 6.8^{a}$ & $65.7 \pm 16.4^{A}$ & $74.6 \pm 10.4^{a, b}$ & $144.3 \pm 41^{A}$ & $169.6 \pm 25.1^{b}$ & $353.0 \pm 22.6 *^{B}$ \\
\hline & n-3/n-6 ratio & $2.5 \pm 0.3^{a}$ & $2.0 \pm 0.1$ & $2.5 \pm 0.2^{a}$ & $2.1 \pm 0.2 * A$ & $3.0 \pm 0.2^{b}$ & $2.8 \pm 0.1 *^{B}$ & $3.3 \pm 0.0^{b}$ & $3.4 \pm 0.0^{c}$ \\
\hline & Total FA & $132.5 \pm 15.2^{a}$ & $321.0 \pm 33.5 * A$ & $161.9 \pm 27.5^{a}$ & $252.3 \pm 54.4 * A$ & $253.2 \pm 36.1^{a}$ & $470.1 \pm 123.3^{A}$ & $525.4 \pm 78.3^{b}$ & $1029.9 \pm 77.0 * B$ \\
\hline \multirow[t]{20}{*}{ (C) } & $14: 0$ & $19.1 \pm 1.1$ & $18.7 \pm 2.1$ & $18.5 \pm 0.7$ & $15.9 \pm 0.4$ & $19.0 \pm 0.3$ & $16.9 \pm 0.6$ & $20.1 \pm 1.4$ & $16.9 \pm 0.5$ \\
\hline & $16: 0$ & $164.1 \pm 3.4$ & $164.4 \pm 13.3$ & $150.6 \pm 0.4$ & $152.2 \pm 1.8$ & $149.1 \pm 9.4$ & $154.4 \pm 2.2$ & $150.8 \pm 5.4$ & $141.0 \pm 4.6$ \\
\hline & $18: 0$ & $92.8 \pm 5.4$ & $106.8 \pm 20.0$ & $87.1 \pm 3.6$ & $102.4 \pm 4.0$ & $85.2 \pm 2.5$ & $99.0 \pm 8.3$ & $101.4 \pm 15.3$ & $99.8 \pm 11.9$ \\
\hline & Total SFA & $276.0 \pm 6.9$ & $289.9 \pm 35.0$ & $256.2 \pm 4.7$ & $270.5 \pm 4.7$ & $253.3 \pm 8.7$ & $270.3 \pm 9.3$ & $272.4 \pm 20.4$ & $257.7 \pm 17.0$ \\
\hline & $16: 1 \mathrm{n}-7$ & $15.3 \pm 0.6$ & $13.5 \pm 0.7$ & $15.2 \pm 0.6$ & $11.7 \pm 0.5^{*}$ & $16.1 \pm 0.8$ & $13.6 \pm 0.5$ & $15.2 \pm 0.9$ & $13.9 \pm 0.5$ \\
\hline & $18: 1 \mathrm{n}-7$ & $13.2 \pm 1.3$ & $10.5 \pm 0.9$ & $12.5 \pm 1.5$ & $10.4 \pm 1.1$ & $13.1 \pm 2.3$ & $11.8 \pm 1.8$ & $12.5 \pm 2.2$ & $10.3 \pm 1.0$ \\
\hline & $18: 1 \mathrm{n}-9$ & $148.4 \pm 6.5$ & $121.0 \pm 4.5^{*}$ & $140.7 \pm 6.5$ & $120.5 \pm 5.2^{*}$ & $143.0 \pm 16.9$ & $136.1 \pm 9.2$ & $135.1 \pm 11.9$ & $119.9 \pm 6.8$ \\
\hline & Total MUFA & $176.9 \pm 7.2$ & $145.1 \pm 5.5 *$ & $168.5 \pm 7.7$ & $142.6 \pm 5.9 *$ & $172.3 \pm 20.0$ & $161.5 \pm 11.4$ & $162.7 \pm 14.9$ & $144.1 \pm 8.2$ \\
\hline & $20: 4 n-3$ & $3.4 \pm 0.1^{\mathrm{a}}$ & $3.0 \pm 0.2 * \mathrm{~A}$ & $3.0 \pm 0.1^{b}$ & $2.6 \pm 0.1 *^{\mathrm{B}}$ & $2.6 \pm 0.3^{\mathrm{a}, \mathrm{b}}$ & $2.5 \pm 0.2^{\mathrm{A}, \mathrm{B}}$ & $2.5 \pm 0.2^{\mathrm{a}, \mathrm{b}}$ & $2.9 \pm 0.5^{\mathrm{A}, \mathrm{B}}$ \\
\hline & $20: 5 n-3$ & $55.1 \pm 2.0^{\mathrm{a}}$ & $46.6 \pm 2.3^{* A}$ & $51.6 \pm 2.2^{\mathrm{a}, \mathrm{b}}$ & $41.3 \pm 1.9^{* \mathrm{~B}}$ & $49.6 \pm 5.1^{\mathrm{a}, \mathrm{b}}$ & $45.5 \pm 2.2^{\mathrm{A}, \mathrm{B}}$ & $47.7 \pm 3.4^{\mathrm{b}}$ & $43.7 \pm 2.0^{\mathrm{A}, \mathrm{B}}$ \\
\hline & $22: 5 n-3$ & $26.6 \pm 0.5$ & $20.7 \pm 1.1 * \mathrm{~A}, \mathrm{~B}$ & $25.5 \pm 0.2$ & $20.9 \pm 0.6^{* A}$ & $25.6 \pm 1.9$ & $23.0 \pm 1.2^{\mathrm{A}, \mathrm{B}}$ & $22.5 \pm 1.3$ & $17.5 \pm 0.1 *^{\mathrm{B}}$ \\
\hline & $22: 6 n-3$ & $58.6 \pm 8.7$ & $44.9 \pm 7.0^{\mathrm{A}}$ & $59.2 \pm 8.4$ & $48.3 \pm 8.6^{\mathrm{A}, \mathrm{B}}$ & $65.5 \pm 13.7$ & $58.8 \pm 11.7^{\mathrm{B}}$ & $62.0 \pm 11.5$ & $49.3 \pm 6.8^{\mathrm{A}, \mathrm{B}}$ \\
\hline & Total n-3 PUFA & $143.7 \pm 10.8$ & $115.2 \pm 9.5 *$ & $139.3 \pm 10.4$ & $113.0 \pm 10.7 *$ & $143.3 \pm 20.9$ & $129.8 \pm 15.1$ & $134.7 \pm 16.3$ & $113.3 \pm 8.7$ \\
\hline & $18: 2 n-6$ & $7.7 \pm 0.5^{\mathrm{a}}$ & $6.9 \pm 0.4$ & $7.0 \pm 0.5^{\mathrm{a}, \mathrm{b}}$ & $6.3 \pm 0.5$ & $6.7 \pm 0.9^{\mathrm{a}, \mathrm{b}}$ & $6.6 \pm 0.7$ & $6.5 \pm 0.8^{b}$ & $5.9 \pm 0.5$ \\
\hline & $20: 3 n-6$ & $5.6 \pm 0.3^{a}$ & $4.8 \pm 0.2^{\mathrm{A}}$ & $4.9 \pm 0.2^{\mathrm{a}, \mathrm{b}}$ & $4.6 \pm 0.3^{A}$ & $4.4 \pm 0.5^{\mathrm{b}, \mathrm{c}}$ & $4.2 \pm 0.3^{\mathrm{A}}$ & $3.9 \pm 0.3^{c}$ & $3.1 \pm 0.1 *^{\mathrm{B}}$ \\
\hline & $20: 4 n-6$ & $24.1 \pm 3.8$ & $24.9 \pm 1.7^{\mathrm{A}}$ & $20.5 \pm 2.6$ & $22.9 \pm 2.8^{* A}$ & $19.0 \pm 3.1$ & $20.8 \pm 2.1^{\mathrm{A}, \mathrm{B}}$ & $17.2 \pm 2.1$ & $14.7 \pm 0.4^{\mathrm{B}}$ \\
\hline & Total n-6 PUFA & $37.4 \pm 4.6^{a}$ & $36.6 \pm 2.3^{A}$ & $32.4 \pm 3.2^{a, b}$ & $33.8 \pm 3.5 * \mathrm{~A}$ & $30.2 \pm 4.5^{\mathrm{a}, \mathrm{b}}$ & $31.6 \pm 3.0^{A}$ & $27.6 \pm 3.1^{b}$ & $23.6 \pm 0.9^{B}$ \\
\hline & Total PUFA & $181.1 \pm 15.3$ & $151.8 \pm 11.7 *$ & $171.7 \pm 13.5$ & $146.8 \pm 14.2 *$ & $173.5 \pm 25.4$ & $161.4 \pm 18.1$ & $162.3 \pm 19.4$ & $137.0 \pm 9.6$ \\
\hline & n-3/n-6 ratio & $3.9 \pm 0.2^{a}$ & $3.1 \pm 0.1 * A$ & $4.3 \pm 0.2^{a, b}$ & $3.4 \pm 0.1 * A$ & $4.8 \pm 0.0^{b, c}$ & $4.1 \pm 0.1 *^{B}$ & $4.9 \pm 0.0^{c}$ & $4.8 \pm 0.2^{C}$ \\
\hline & Total FA & $634.0 \pm 22.5$ & $586.8 \pm 29.8$ & $596.4 \pm 16.5$ & $559.9 \pm 16.5 *$ & $599.1 \pm 52.9$ & $593.3 \pm 21.8$ & $597.4 \pm 19.2$ & $538.7 \pm 3.0$ \\
\hline
\end{tabular}

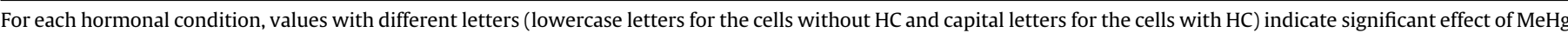

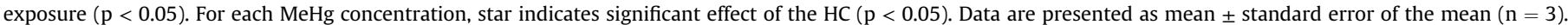

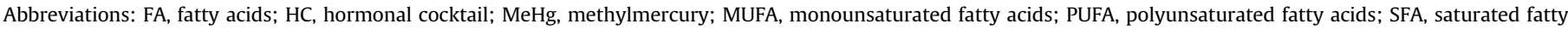
acids; $\mathrm{THg}$, total mercury. 


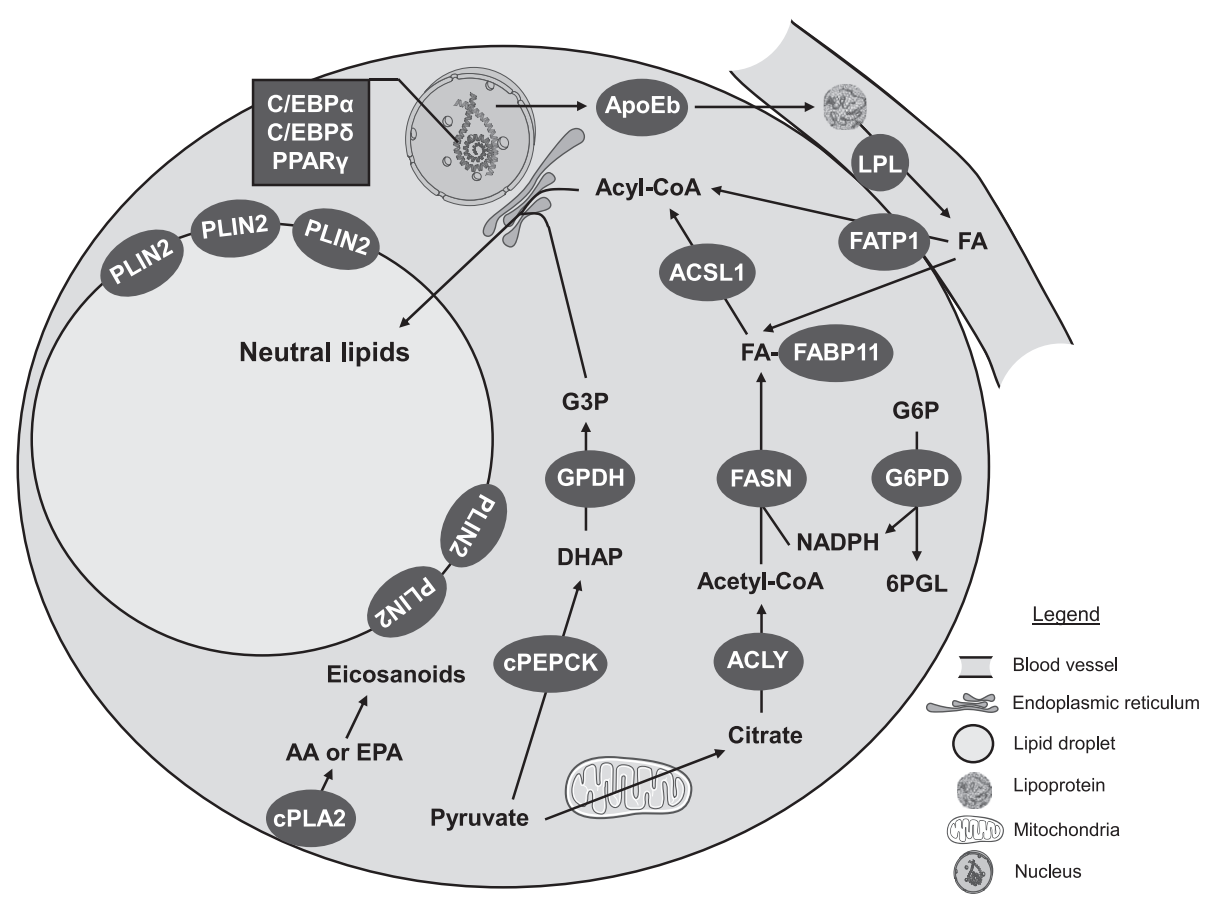

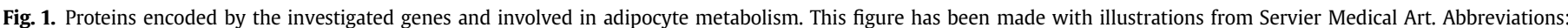

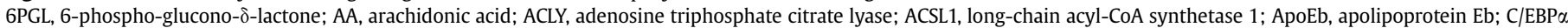

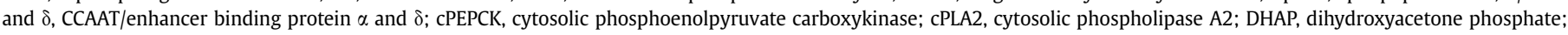

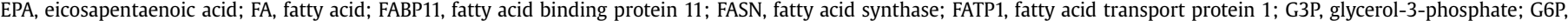

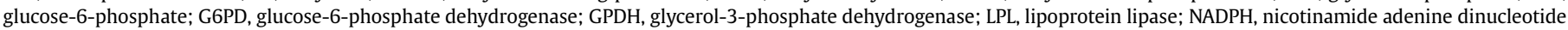
phosphate; PLIN2, perilipin 2; PPAR $\gamma$, peroxisome proliferator-activated receptor $\gamma$.

days) (Liu et al., 2013), in muscle, brain and liver of zebrafish (MeHg, 5 and $13.5 \mu \mathrm{g} / \mathrm{g}, 63$ days) (Gonzalez et al., 2005) and in holothurians $\left(\mathrm{HgCl}_{2}, 40,80\right.$ and $160 \mu \mathrm{g} / \mathrm{L}, 4$ days) (Telahigue et al., 2019). Mechanisms for MeHg uptake have been suggested to include both passive and (specific and non-specific) active transports (Kidd and Batchelar, 2011). More specifically, uptake of MeHg-chloride seems to occur via passive diffusion and uptake of MeHg-L-cysteine via a carrier protein (Heggland et al., 2009).

In the present study, as the culture medium contained L-cysteine ( $1 \mathrm{mM}$ ) and as MeHg has a higher affinity for sulfhydryl groups than for chloride ions (reviewed by Nogara et al. (2019)), MeHg was most likely transported via membrane transport protein under the form of MeHg-L-cysteine complex. A plausible transporter candidate is the system L-type large neutral amino acid transporter (LAT) (Heggland et al., 2009). This transporter is $\mathrm{Na}^{+}$-independent obligatory exchanger (Verrey, 2003). The expression of the corresponding gene was found in rat adipose tissue (isoforms LAT1 and LAT2) (Ritchie et al., 2001) and in 3T3-L1 cells (only LAT1 isoform) (Mitchell et al., 2010). An equivalent transporter called solute carrier family member 43 (SLC 43) was also found in various teleost fish (Gallardo et al., 1996; Verri et al., 2012; Barat et al., 2019).

Between 9 and 14\% of MeHg added throughout the culture were found accumulated in cells at the end of the experimental period. The relatively low amount of $\mathrm{MeHg}$ accumulated in preadipocytes may be explained by a low bioavailability of $\mathrm{MeHg}$ resulting from its tendency to bind to sulfhydryl groups (e.g. from molecules of FBS) (Rabenstein and Fairhurst, 1975). In addition, the presence of other substrates of LAT1/2 such as L-methionine, L-leucine and Lhistidine in the culture medium could reduce the MeHg-L-cysteine uptake by this carrier as observed in C6 rat glioma and RBE4 rat brain endothelial cell lines (Heggland et al., 2009). The fairly low accumulation of $\mathrm{MeHg}$ by preadipocytes contrasts with the case of other, more lipid-soluble, environmental pollutants such as PCBs, which have been shown to massively accumulate within a few hours in cultures of rodent adipocytes (Bourez et al., 2012b, 2013). Those distinct in vitro behaviors are in accordance with the fact that adipose tissue does not represent an important reservoir for $\mathrm{MeHg}$, as opposed to PCBs (Wagemann et al., 1998; Covaci et al., 2008).

\subsubsection{Increased content in neutral lipids following $\mathrm{MeHg}$ and $\mathrm{HC}$ treatments}

The highest MeHg concentration ( $3.8 \mu \mathrm{M})$ sharply increased the level of neutral lipids in preadipocytes in both hormonal conditions $(+296 \%$ and $+220 \%$ in absence and presence of $\mathrm{HC}$, respectively) (Table 1-B). As observed in cells non-exposed to MeHg, $\mathrm{HC}$ induced a significant (except at $1.7 \mu \mathrm{M} \mathrm{MeHg}$ ) increase in the total neutral lipid content $(+56 \%,+86 \%$ and $+96 \%$ at $0.3,1.7$ and $3.8 \mu \mathrm{M} \mathrm{MeHg}$, respectively) (Table $1-\mathrm{B}$ ). The combined exposure to both factors exhibited an impact on fatty acid accumulation that was greater than additive. Indeed, the total fatty acid content was increased by $142 \%$ by the $\mathrm{HC}, 296 \%$ by the $\mathrm{MeHg}$, and $677 \%$ by the combination of HC and $\mathrm{MeHg}$, indicating an effect that was 54\% greater than additive. This result highlights a synergism between the effects of $\mathrm{MeHg}$ and HC. Contrasting results were found in the literature regarding the effect of mercury on lipid accumulation. In human preadipocytes and mesenchymal stem cells, $\mathrm{MeHg}$ (1, 10 and $100 \mathrm{nM}, 11$ days) did not affect lipid accumulation (van den Dungen et al., 2017). Note that the concentrations used were lower than in the present study. In contrast, a phenotype similar to the one observed herein was reported in liver of $\mathrm{HgCl}_{2}$-treated zebrafish (50 

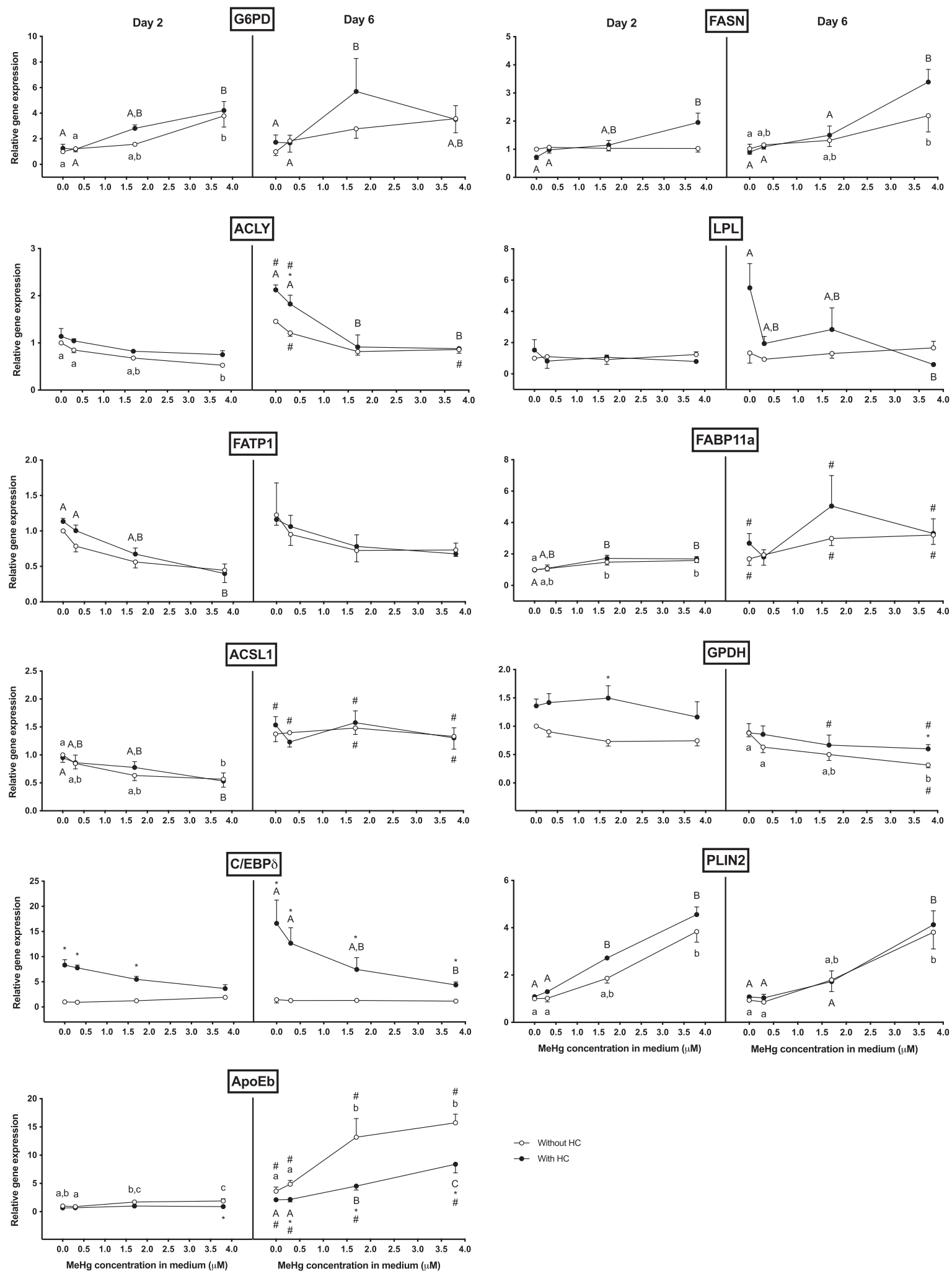

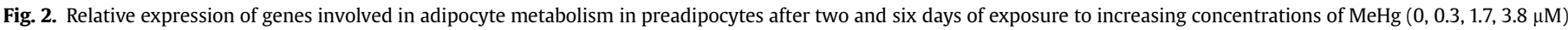

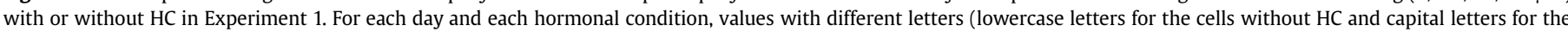

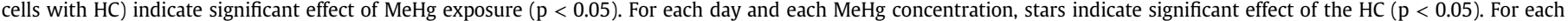

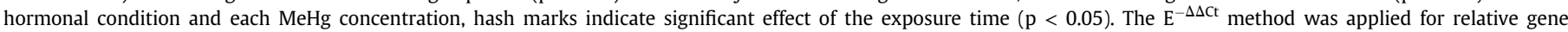

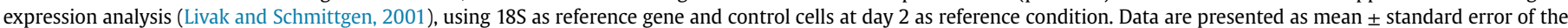

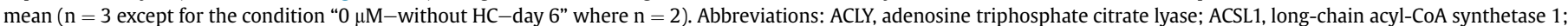

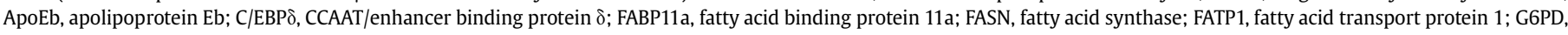
glucose-6-phosphate dehydrogenase; GPDH, glycerol-3-phosphate dehydrogenase; HC, hormonal cocktail; LPL, lipoprotein lipase; MeHg, methylmercury; PLIN2, perilipin 2. 
and $200 \mu \mathrm{g} / \mathrm{L}, 96 \mathrm{~h}$ ), with increased number and size of lipid droplets (Ung et al., 2010). Contrary to neutral lipids, the 6-day exposure to MeHg did not modify the total amount of phospholipids in preadipocytes (Table $1-\mathrm{C}$ ). Similarly, none of the $\mathrm{MeHg}$ concentrations had an effect on the amount of cellular proteins in this study (data not shown).

\subsubsection{Effects of $\mathrm{MeHg}$ on lipid metabolism at transcriptional level}

The expression level of several genes was assessed to investigate their potential involvement in the effects of $\mathrm{MeHg}$ on neutral lipid accumulation. The expression of glucose-6-phosphate dehydrogenase (G6PD) was significantly increased at day 2 by MeHg exposure in both hormonal conditions (Fig. 2). G6PD generates nicotinamide adenine dinucleotide phosphate (NADPH) through the pentose phosphate pathway (Fig. 1). NADPH can be used for the de novo synthesis of fatty acids via the fatty acid synthase (FASN). In the present study, the expression of FASN was significantly upregulated by the highest $\mathrm{MeHg}$ concentration at day 2 in presence of HC as well as at day 6 in both hormonal conditions (Fig. 2) and could partially explain the accumulation of lipids observed herein. In in vivo situation, a decrease of FASN expression was, however, observed in the liver of fathead minnow exposed to $\mathrm{MeHg}$ $(2 \mu \mathrm{g} / \mathrm{g}, 96 \mathrm{~h})$ (Klaper et al., 2008). On the other hand, the liver transcriptome of $\mathrm{HgCl}_{2}$-treated zebrafish revealed the upregulation of fatty acid synthesis pathway within $96 \mathrm{~h}$ without, however, specifying the enzyme(s) involved (Ung et al., 2010). Triphenyltin (TPT), a well-known obesogen, also induced an increased expression of FASN in trout preadipocytes (Lutfi et al., 2017). On the other hand, after two and six days of contamination, a significant and negative impact of $\mathrm{MeHg}$ was observed on the expression of ACLY, another enzyme involved in de novo fatty acid synthesis pathway (Fig. 1), in absence and presence of $\mathrm{HC}$ (Fig. 2). In Atlantic cod, MeHg also down regulated ACLY in liver at the protein level (Yadetie et al., 2016).

Triglycerides can also be composed of exogenous fatty acids taken up from blood (in vivo) or culture medium (in vitro). The lipoprotein lipase (LPL) hydrolyses triglycerides from circulating lipoproteins (Fig. 1). This enzyme is produced by adipocytes and is considered as an early marker of adipocyte differentiation (Bouraoui et al., 2012). Fatty acids are then transported across the membrane by passive diffusion or via fatty acid transport proteins (FATPs) and in the cytosol via FABPs (Fig. 1). In the present study, MeHg negatively affected the expression of LPL only at day 6 and in presence of HC (Fig. 2). As observed herein after two days, the expression of LPL was not impacted after a 24-h exposure to TBT or TPT in trout preadipocytes, suggesting the low implication of this enzyme in the short-term toxicity of lipid metabolism disruptors (Lutfi et al., 2017). MeHg significantly down-regulated the expression of FATP1 at day 2 in presence of HC (Fig. 2). A reduction in the expression of FATP1 by MeHg was also observed in RTL-W1 cell line (Ferain et al., 2018). The MeHg exposure slightly but significantly increased the expression of FABP11a at day 2 in both hormonal conditions (Fig. 2). Similar results were obtained for FABP11b (data not shown). Note that the FABP11a isoform was highly expressed in preadipocytes as evidenced by the low $\mathrm{Ct}$ values (data not shown). The expression of another isoform (FABP10) was also up-regulated in the muscle of MeHg-contaminated zebrafish (3 nmol Hg/day/ fish, 25 days) (Cambier et al., 2009). The up-regulation of FABP11a by $\mathrm{MeHg}$ reflects an increased need for intracellular fatty acid transport. This effect could contribute to a higher transfer of fatty acids towards lipid droplets.

After transport, fatty acids can be activated to acyl-CoA by an acyl-CoA synthetase (Fig. 1). The exposure to $\mathrm{MeHg}$ significantly reduced the expression of ACSL1 at day 2 in both hormonal conditions (Fig. 2). However, the effect disappeared at day 6 .
The triglyceride synthesis also needs glycerol-3-phosphate as carbon backbone produced from dihydroxyacetone phosphate by the glycerol-3-phosphate dehydrogenase (GPDH) (Fig. 1). The highest MeHg concentration decreased the expression of GPDH at day 6 in absence of HC. On the other hand, no experimental condition exerted a significant effect on the expression of cytosolic phosphoenolpyruvate carboxykinase (CPEPCK), another enzyme involved in glyceroneogenesis (Fig. 1) (data not shown). Accordingly, the impact of MeHg on neutral lipid content does not seem to involve the expression of glyceroneogenesis-related genes.

\subsubsection{Modulation of adipocyte differentiation by $\mathrm{MeHg}$}

The lipid accumulation observed in contaminated preadipocytes could be concurrent with the induction of adipogenesis. The expression of several genes specifically transcribed during the adipocyte differentiation was therefore evaluated. $\mathrm{MeHg}$ downregulated (only significantly at day 6) the HC-induced expression of $\mathrm{C} / \mathrm{EBP} \delta$, whereas no effect of MeHg was observed in absence of $\mathrm{HC}$ (Fig. 2). Opposite results were found in $\mathrm{HgCl}_{2}$-treated zebrafish liver, where the expression of C/EBP $\delta$ and $\beta$ in the liver was upregulated by mercury (Ung et al., 2010). This difference highlights potential tissue-dependent response to contamination as well as the fact that organic and inorganic mercury can act via different mechanisms, especially at the gene expression level, as suggested by McElwee et al. (2013). The expression of two other key transcription factors, C/EBP $\alpha$ and PPAR $\gamma$, yet involved in adipogenesis in numerous vertebrate species (Rosen et al., 2002), including rainbow trout (Bou et al., 2017), was not impacted by the $\mathrm{MeHg}$ exposure (data not shown). Contradictory results were found in literature regarding the effects of mercury on these transcription factors. In 3T3-L1 cell line, inorganic mercury significantly decreased the level of PPAR $\gamma$ protein $(5$ and $10 \mu \mathrm{M}, 48 \mathrm{~h}$ ) (Barnes et al., 2003) whereas the PPAR $\gamma$ mRNA level increased in $\mathrm{Hg}$ exposed rats (first dose of $4.6 \mu \mathrm{g} / \mathrm{kg}$ and subsequent doses of $0.07 \mu \mathrm{g} / \mathrm{kg} / \mathrm{day}, 60$ days) (Rizzetti et al., 2019). The implication of these transcription factors in the toxicity of mercury requires further research. The exposure of preadipocytes to MeHg substantially increased the expression of perilipin 2 (PLIN2), a protein synthesized by adipocytes and present at the surface of lipid droplets, in both hormonal conditions at day 2 and day 6 (Fig. 2). PLIN2 mRNA and protein levels have been shown to be higher during the adipocyte differentiation than during the proliferation phase (Bou et al., 2017), highlighting the implication of this protein in the adipocyte maturation process (Sztalryd and Kimmel, 2014; Bou et al., 2017) and suggesting a potential role in the $\mathrm{MeHg}$ toxicity. ApoE is a component of circulating lipoproteins synthesized inter alia by adipocytes. In the present study, MeHg (1.7 and $3.8 \mu \mathrm{M})$ significantly up-regulated its expression at day 6 in both hormonal conditions while the presence of HC significantly downregulated the effect of the $\mathrm{MeHg}$ (Fig. 2). The expression of ApoEb was also up-regulated in the muscle of MeHg-contaminated zebrafish (Cambier et al., 2009). Previous studies in mice adipocytes revealed that adipocyte-synthesized ApoE facilitates intracellular triglyceride accumulation (Huang et al., 2006, 2009; Li and Liu, 2014).

As previously mentioned, the expression of G6PD was upregulated by $\mathrm{MeHg}$ at day 2. It could reflect the possible growing need for reducing equivalents to cope with the oxidative stress generated by $\mathrm{MeHg}$, in addition to supply fatty acid de novo synthesis. Indeed, $\mathrm{MeHg}$ is known to increase the level of reactive oxygen species (ROS) in organisms or cultured cells (Farina et al., 2011; Ynalvez et al., 2016). Although contradictory results are found in the literature, the majority of recent studies in mouse, rat and human models attributed a pro-adipogenic role to ROS, especially in the beginning of the differentiation process (De Villiers et al., 2017). In 
Atlantic salmon, oxidative stress negatively modulated the terminal differentiation of preadipocytes (Todorčević et al., 2010). The global impact of ROS on preadipocyte therefore seems to depend on the stage of differentiation of the cells.

\subsubsection{Modulation of neutral lipid fatty acid composition by $\mathrm{MeHg}$}

The concentrations of all fatty acids except 22:1 n-9 were significantly higher at $3.8 \mu \mathrm{M}$ MeHg than at lower MeHg concentrations ( 0 and $0.3 \mu \mathrm{M} \mathrm{MeHg}$ in absence of $\mathrm{HC}$ and $0,0.3$ and $1.7 \mu \mathrm{M}$ $\mathrm{MeHg}$ in presence of $\mathrm{HC}$ ) (Table 1-B). Nevertheless, the level of increase was not the same for all fatty acid classes. The total n-3 polyunsaturated fatty acids (PUFA) showed the highest level of increase $(+584 \%$ and $+396 \%$ in presence and absence of $\mathrm{HC}$, respectively) followed by the total n-6 PUFA $(+417 \%$ and $+188 \%)$ and the total monounsaturated fatty acids (MUFA) content $(+346 \%$ and $+222 \%$ ). The total saturated fatty acids (SFA) content showed the lowest increase $(+147 \%$ and $+142 \%)$. When data are expressed in terms of proportions, the highest increase was also observed for n-3 PUFA, while the proportions of SFA dropped in both hormonal conditions (Table A.3-A). As a result of the preferential accumulation of n-3 PUFA, the n-3/n-6 ratio increased in a dose-dependent manner in both hormonal conditions. In the literature, contamination of holothurians with $\mathrm{HgCl}_{2}$ induced an increase of the proportion of n-3 PUFA, especially docosahexaenoic acid (DHA, 22:6 n3 ), and a decrease of n-6 PUFA (Telahigue et al., 2019). The proportion of total SFA also increased although the proportion of 16:0, the most abundant SFA in preadipocytes, decreased at the highest doses of inorganic mercury in sea cucumber (Telahigue et al., 2019). Note that this study analysed the fatty acid composition of the total lipids, possibly hiding lipid fraction-dependent effects.

\subsubsection{Modulation of phospholipid fatty acid composition by $\mathrm{MeHg}$}

MeHg significantly affected the membrane phospholipids fatty acid profile (Table $1-\mathrm{C}$ ). Especially, the n-6 PUFA content greatly decreased after exposure to $3.8 \mu \mathrm{M} \mathrm{MeHg}(-26 \%$ in absence and $-35 \%$ in presence of HC compared to non-exposed cells). The highest drop of concentrations could be noticed for 18:2 n-6, 20:3 n-6 and arachidonic acid (AA, 20:4 n-6). No difference in the total n3 PUFA content was observed after MeHg contamination. However, eicosapentaenoic acid (EPA, 20:5 n-3) concentration was significantly lower in cells exposed to $3.8 \mu \mathrm{M}$ MeHg as compared to control cells. On the other hand, DHA concentration was significantly higher in cells exposed to $1.7 \mu \mathrm{M}$ MeHg as compared to nonexposed cells in presence of HC. As a result of the n-6 PUFA depletion, the n-3/n-6 ratio significantly increased in the membranes of preadipocytes exposed to $3.8 \mu \mathrm{M}$ MeHg. No modification was observed regarding SFA and MUFA contents. In terms of proportions, comparable results were obtained especially regarding the effects of MeHg on the n-6 PUFA and DHA (Table A.3-B). Similar results were found in the literature concerning the $n-6$ PUFA. The proportions of $18: 2 n-6,20: 3 n-6$ and AA also decreased in the Hgcontaminated sea cucumber body wall (Telahigue et al., 2019) and tended to decrease in phospholipids of AA-enriched RTL-W1 cells after exposure to MeHg (Ferain et al., 2018). The reduction of AA from membrane phospholipids was also observed in phospholipids of Atlantic salmon brain after chronic dietary exposure to $\mathrm{MeHg}$ (5 mg/kg, 3 months) (Amlund et al., 2012), in primary rat neurons (Shanker et al., 2004) and astrocytes (Shanker et al., 2002), and in bovine pulmonary artery endothelial cell line (Mazerik et al., 2007; Sherwani et al., 2013) (5 $\mu \mathrm{M}$ MeHg, 0-120 min). Concerning the n-3 PUFA in phospholipids, Olsvik et al. (2011) also showed a reduced content of EPA in Atlantic salmon brain after a 3-month exposure to MeHg (5 mg/kg) and Telahigue et al. (2019) showed a lower proportion of EPA and a higher proportion of DHA after $\mathrm{HgCl}_{2}$ contamination. Fatty acids with twenty carbons, particularly AA and EPA, are known to be released from phospholipids by phospholipases (mainly cytosolic phospholipase A2 (cPLA2)) to provide precursors of eicosanoids which are involved in various biological processes like inflammation (Kremmyda et al., 2011). The reduction of AA and EPA could be related to an increased activity and/or expression of cPLA2 (Shanker et al., 2004). In our experiments, the expression of CPLA2 was however not influenced by $\mathrm{MeHg}$ (data not shown). Similarly, no modification of cPLA2 mRNA level was observed in MeHg-treated RTL-W1 cells (Ferain et al., 2018). However, such results do not exclude the possibility that $\mathrm{MeHg}$ exerts an impact on CPLA2 at a post-transcriptional level.

\subsection{Preadipocytes exposed to $\mathrm{MeHg}$ and HC for increasing durations (Experiment 2)}

\subsubsection{No time-dependent accumulation of $\mathrm{MeHg}$ within days}

In the second experiment, preadipocytes were contaminated with $3.8 \mu \mathrm{M} \mathrm{MeHg}$ during three exposure durations (two, four and six days). After two days, the percentage of $\mathrm{MeHg}$ accumulation by the cells was $39 \%$ of the total MeHg amount added in the culture medium. As presented above, this percentage was much lower after six days (around 10\%). The difference can be explained by the fact that no additional cellular $\mathrm{MeHg}$ accumulation occurred between day 2 and day 6 of exposure, even if $\mathrm{MeHg}$ was still added in the medium. Indeed, the intracellular mercury concentration did not differ between two, four or six days of exposure (Fig. 3, black bars). A similar behaviour was observed in PC12 cells exposed to $10 \mathrm{nM}$ MeHg for six days (Meacham et al., 2005). The present study reveals that the retention limit of preadipocytes for $\mathrm{MeHg}$ is fairly quickly reached, as opposed to other more fat-soluble pollutants like PCBs that can be accumulated at extremely high concentrations in cultured fat cells (Bourez et al., 2012a).

\subsubsection{Release of $\mathrm{THg}$ accumulated in cells when $\mathrm{MeHg}$ exposure stops}

Cells exposed to MeHg during only two or four days were thereafter maintained in a MeHg-free medium until day 6. The $\mathrm{THg}$ concentration in these cells at day 6 significantly dropped compared to the concentrations measured at days 2 and 4 (Fig. 3 , dark grey bars). Indeed, $95 \%$ and $79 \%$ of the $\mathrm{THg}$ accumulated in preadipocytes at days 2 and 4 respectively, were released into the culture medium at day 6 (Fig. 3, light grey bars). For both durations, the sum of the THg concentration measured at day 6 in cells and in medium equaled the intracellular THg concentration at day 2 or 4 . Such phenomenon suggests an efficient efflux of $\mathrm{MeHg}$ from preadipocytes. MeHg efflux was studied in C6 and RBE4 cell lines and LAT1/2 did not seem to be involved in this process (Kerper et al., 1996; Heggland et al., 2009). The presence of L-cysteine $(100 \mu \mathrm{M})$ and L-cystine $(1 \mathrm{mM})$ in the culture medium has been shown to increase the MeHg efflux and to lead to an almost complete depletion of intracellular $\mathrm{MeHg}$ after $60 \mathrm{~min}$ in these cell lines (Heggland et al., 2009). The authors suggested that the high extracellular concentration of cysteine and cystine induces an increase of the intracellular concentration of cysteine and, as a consequence, the synthesis of glutathione (GSH), cysteine being in most cases the limiting amino acid in this pathway (Wu et al., 2004). The MeHg-GSH complex generated by exchange reaction could then be transported out of the cells. Additional experiments are however required to validate such mechanism of efflux in preadipocytes.

\subsubsection{Accumulation of neutral lipids persisted after the onset of mercury release by the cells}

The cells exposed to MeHg during four or six days stored similar 


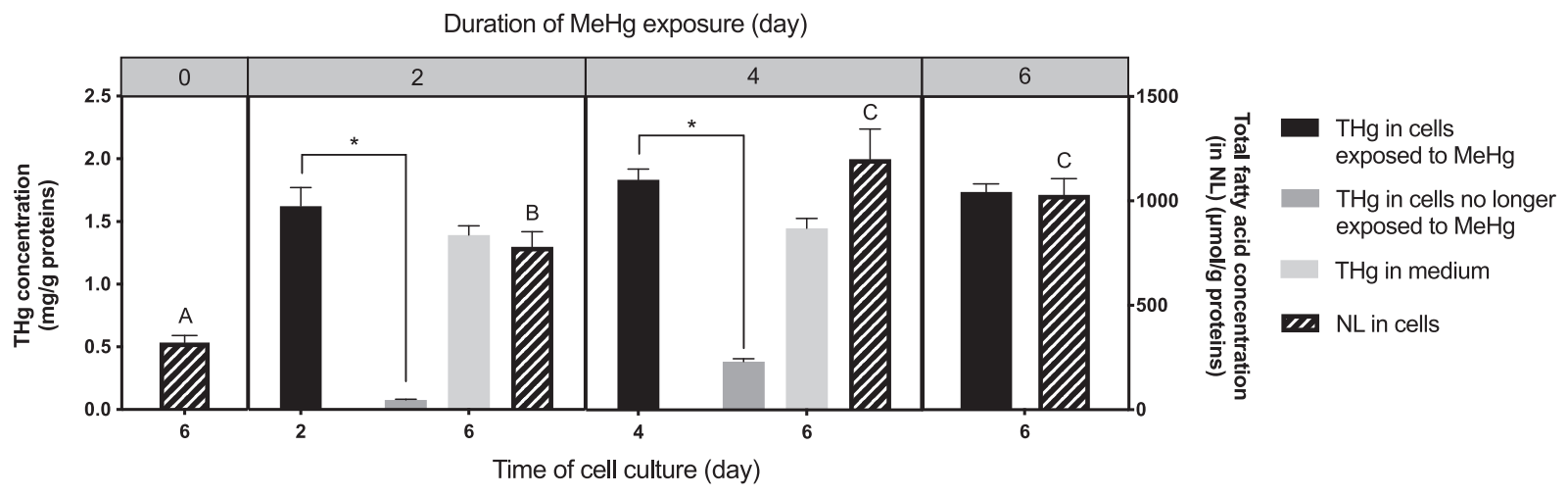

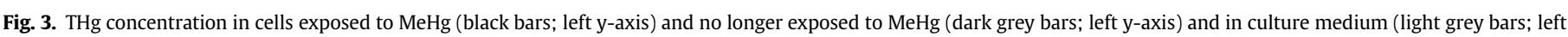

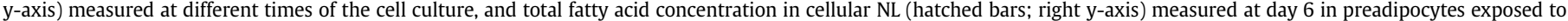

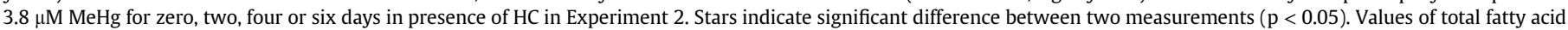

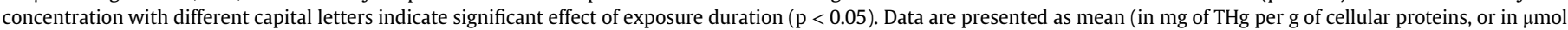

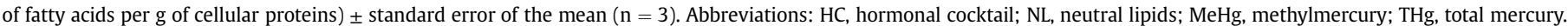

amounts of neutral lipids at day 6 (Fig. 3, hatched bars), which suggests that the effect induced by $\mathrm{MeHg}$ on the intracellular pool of lipids continued beyond the initiation of intracellular THg drop. As previously described, this lipid storage might be associated with the adipocyte differentiation induced by the production of ROS as well as the upregulation of lipogenic genes. A recent study showed that chronic mercury contamination $\left(\mathrm{HgCl}_{2}\right.$, first dose of $4.6 \mu \mathrm{g} / \mathrm{kg}$ and subsequent doses of $0.07 \mu \mathrm{g} / \mathrm{kg} / \mathrm{day}, 60$ days) impaired rat epididymal white adipose tissue metabolism even though the mercury concentration in the adipose tissue of treated rats was not significantly different from untreated animals (Rizzetti et al., 2019). The authors suggested the implication of the oxidative and endoplasmic reticulum stresses in adipose tissue. Moreover, an offset between $\mathrm{MeHg}$ exposure and the appearance of MeHg-related adipose disruption is often observed. In a recent in vivo study, it has been shown that the exposure of female rats to cadmium and $\mathrm{MeHg}$ at the periconception period, when adipose tissue is differentiating, led to adult male offspring with higher body and abdominal adipose tissue weights (Camsari et al., 2016). Similarly, in the present study, preadipocytes were exposed to MeHg as early as the induction of adipocyte differentiation. As explained above, the moment of MeHg exposure seems to be a critical element to determine the potential future effects.

\section{Conclusion}

In summary, environmentally relevant $\mathrm{MeHg}$ concentrations exerted pro-adipogenic effects on rainbow trout preadipocytes. A dose-dependent accumulation of neutral lipids (mainly n-3 PUFA) was observed even after the onset of mercury release by the cells. The accumulation of lipids could be explained in part by an increase in the expression of FABP11a and FASN. Adipocyte-related genes (PLIN2 and ApoEb) were positively modulated by $\mathrm{MeHg}$ and could also contribute to adipogenic properties of the organo-metal. Further experiments are however required to better understand the impacts of MeHg on the adipogenesis process and lipid metabolism but this study provides evidence to consider $\mathrm{MeHg}$ as an actor in the current obesity pandemic.

\section{Credit author statement}

Gilles Tinant: Conceptualization, Formal analysis, Investigation, Methodology, Visualization, Roles/Writing - original draft ; Ineke Neefs: Investigation ; Krishna Das: Resources ; Jean-François Rees:
Funding acquisition, Supervision ; Yvan Larondelle: Funding acquisition, Supervision, Writing - review \& editing ; Cathy Debier: Conceptualization, Funding acquisition, Methodology, Supervision, Visualization, Writing - review \& editing.

\section{Declaration of competing interest}

The authors declare that they have no known competing financial interests or personal relationships that could have appeared to influence the work reported in this paper.

\section{Acknowledgments}

This study was funded by Belspo (IAP AQUASTRESS P7/31) and by the Fonds de la Recherche Scientifique (FNRS). Gilles Tinant is a F.R.I.A. Grant Holder of the FNRS. Krishna Das is a Senior F.R.S.-FNRS Research Associate. The authors acknowledge Catherine Rasse and Vincent Bremhorst for their valuable help in statistical analyses, and Renzo Biondo, France Damseaux and Marianna Pinzonne for their significant help in mercury analyses.

\section{Appendix A. Supplementary data}

Supplementary data to this article can be found online at https://doi.org/10.1016/j.chemosphere.2020.127917.

\section{References}

Amlund, H., Andreasen, L., Torstensen, B.E., 2012. Dietary methylmercury and vegetable oil affects brain lipid composition in Atlantic salmon (Salmo salar L.). Food Chem. Toxicol. 50, 518-525.

Ariemma, F., D’Esposito, V., Liguoro, D., Cabaro, S., Liotti, A., Cimmino, I., Longo, M., Beguinot, F., Formisano, P., Valentino, R., 2016. Low-dose bisphenol-A impairs adipogenesis and generates dysfunctional 3T3-L1 adipocytes. PloS One 11, e0150762.

Barat, A., Sahoo, P.K., Kumar, R., Goel, C., Siva, C., Ali, S., 2019. Data on solute carrier transporter genes of a threatened Himalayan fish species-Schizothorax richardsonii. Data in Brief 23, 103712.

Barnes, D.M., Hanlon, P.R., Kircher, E.A., 2003. Effects of inorganic $\mathrm{HgCl} 2$ on adipogenesis. Toxicol. Sci. 75, 368-377.

Bjørklund, G., Dadar, M., Mutter, J., Aaseth, J., 2017. The toxicology of mercury: current research and emerging trends. Environ. Res. 159, 545-554.

Bligh, E.G., Dyer, W.J., 1959. A rapid method of total lipid extraction and purification. Can. J. Biochem. Physiol. 37, 911-917.

Bou, M., Montfort, J., Le Cam, A., Rallière, C., Lebret, V., Gabillard, J.-C., Weil, C., Gutiérrez, J., Rescan, P.-Y., Capilla, E., 2017. Gene expression profile during proliferation and differentiation of rainbow trout adipocyte precursor cells. BMC Genom. 18, 347.

Bouraoui, L., Cruz-Garcia, L., Gutiérrez, J., Capilla, E., Navarro, I., 2012. Regulation of lipoprotein lipase gene expression by insulin and troglitazone in rainbow trout 
(Oncorhynchus mykiss) adipocyte cells in culture. Comp. Biochem. Physiol. Mol. Integr. Physiol. 161, 83-88.

Bouraoui, L., Gutiérrez, J., Navarro, I., 2008. Regulation of proliferation and differentiation of adipocyte precursor cells in rainbow trout (Oncorhynchus mykiss). J. Endocrinol. 198, 459-469.

Bourez, S., Joly, A., Covaci, A., Remacle, C., Larondelle, Y., Schneider, Y.-J., Debier, C., 2012a. Accumulation capacity of primary cultures of adipocytes for PCB-126: influence of cell differentiation stage and triglyceride levels. Toxicol. Lett. 214, $243-250$.

Bourez, S., Le Lay, S., Van den Daelen, C., Louis, C., Larondelle, Y., Thomé, J.-P., Schneider, Y.-J., Dugail, I., Debier, C., 2012b. Accumulation of polychlorinated biphenyls in adipocytes: selective targeting to lipid droplets and role of caveolin-1. PLoS One 7, e31834.

Bourez, S., Van den Daelen, C., Le Lay, S., Poupaert, J., Larondelle, Y., Thomé, J.-P., Schneider, Y.-J., Dugail, I., Debier, C., 2013. The dynamics of accumulation of PCBs in cultured adipocytes vary with the cell lipid content and the lipophilicity of the congener. Toxicol. Lett. 216, 40-46.

Cambier, S., Gonzalez, P., Durrieu, G., Maury-Brachet, R., Boudou, A., Bourdineaud, J.P. 2009. Serial analysis of gene expression in the skeletal muscles of zebrafish fed with a methylmercury-contaminated diet. Environ. Sci. Technol. 44, 469-475.

Camsari, C., Folger, J.K., McGee, D., Bursian, S.J., Wang, H., Knott, J.G., Smith, G.W., 2016. Effects of periconception cadmium and mercury co-administration to mice on indices of chronic diseases in male offspring at maturity. Environ. Health Perspect. 125, 643-650.

Chauhan, S., Dunlap, K., Duffy, L.K., 2019. Effects of methylmercury and theaflavin digallate on adipokines in mature 3T3-L1 adipocytes. Int. J. Mol. Sci. 20, 2755.

Covaci, A., Voorspoels, S., Roosens, L., Jacobs, W., Blust, R., Neels, H., 2008. Polybrominated diphenyl ethers (PBDEs) and polychlorinated biphenyls (PCBs) in human liver and adipose tissue samples from Belgium. Chemosphere 73, $170-175$.

Darbre, P.D., 2017. Endocrine disruptors and obesity. Current obesity reports 6, $18-27$.

De Villiers, D., Potgieter, M., Ambele, M.A., Adam, L., Durandt, C., Pepper, M.S., 2017. The role of reactive oxygen species in adipogenic differentiation. In: Stem Cells: Biology and Engineering. Springer, pp. 125-144.

Farina, M., Rocha, J.B., Aschner, M., 2011. Mechanisms of methylmercury-induced neurotoxicity: evidence from experimental studies. Life Sci. 89, 555-563.

Ferain, A., Bonnineau, C., Neefs, I., Das, K., Larondelle, Y., Rees, J.-F., Debier, C., Lemaire, B., 2018. Transcriptional effects of phospholipid fatty acid profile on rainbow trout liver cells exposed to methylmercury. Aquat. Toxicol. 199, $174-187$.

Ferrante, M.C., Amero, P., Santoro, A., Monnolo, A., Simeoli, R., Di Guida, F., Raso, G.M., Meli, R., 2014. Polychlorinated biphenyls (PCB 101, PCB 153 and PCB 180) alter leptin signaling and lipid metabolism in differentiated 3T3-L1 adipocytes. Toxicol. Appl. Pharmacol. 279, 401-408.

Gallardo, M., Albi, J., Sanchez, J., 1996. Uptake of L-leucine by trout red blood cells and peripheral lymphocytes. J. Membr. Biol. 152, 57-63.

Gonzalez, P., Dominique, Y., Massabuau, J., Boudou, A., Bourdineaud, J., 2005. Comparative effects of dietary methylmercury on gene expression in liver, skeletal muscle, and brain of the zebrafish (Danio rerio). Environ. Sci. Technol. 39, 3972-3980.

Grün, F., Blumberg, B., 2009. Minireview: the case for obesogens. Mol. Endocrinol. 23, $1127-1134$.

Habran, S., Pomeroy, P.P., Debier, C., Das, K., 2013. Changes in trace elements during lactation in a marine top predator, the grey seal. Aquat. Toxicol. 126, 455-466.

Harwood Jr., H.J., 2012. The adipocyte as an endocrine organ in the regulation of metabolic homeostasis. Neuropharmacology 63, 57-75.

Heggland, I., Kaur, P., Syversen, T., 2009. Uptake and efflux of methylmercury in vitro: comparison of transport mechanisms in C6, B35 and RBE4 cells. Toxicol. Vitro 23, 1020-1027.

Heindel, J.J., Blumberg, B., 2018. Environmental obesogens: mechanisms and controversies. Annu. Rev. Pharmacol. Toxicol.

Huang, Z.H., Gu, D., Mazzone, T., 2009. Role of adipocyte-derived apoE in modulating adipocyte size, lipid metabolism, and gene expression in vivo. Am. J. Physiol. Endocrinol. Metab. 296, E1110-E1119.

Huang, Z.H., Reardon, C.A., Mazzone, T., 2006. Endogenous ApoE expression modulates adipocyte triglyceride content and turnover. Diabetes 55, 3394-3402.

Kawakami, T. Hanao, N., Nishiyama, K. Kadota, Y. Inoue, M. Sato, M., Suzuki, S., 2012. Differential effects of cobalt and mercury on lipid metabolism in the white adipose tissue of high-fat diet-induced obesity mice. Toxicol. Appl. Pharmacol. 258, 32-42.

Kerper, L.E., Mokrzan, E.M., Clarkson, T.W., Ballatori, N., 1996. Methylmercury efflux from brain capillary endothelial cells is modulated by intracellular glutathione but not ATP. Toxicol. Appl. Pharmacol. 141, 526-531.

Kidd, K., Batchelar, K., 2011. 5 - mercury. Fish Physiol. 237-295.

Klaper, R., Carter, B.J., Richter, C., Drevnick, P., Sandheinrich, M., Tillitt, D., 2008. Use of a $15 \mathrm{k}$ gene microarray to determine gene expression changes in response to acute and chronic methylmercury exposure in the fathead minnow Pimephales promelas Rafinesque. J. Fish. Biol. 72, 2207-2280.

Kremmyda, L.-S., Tvrzicka, E., Stankova, B., Zak, A., 2011. Fatty acids as biocompounds: their role in human metabolism, health and disease-a review. Part 2: fatty acid physiological roles and applications in human health and disease. Biomed. Pap. Med. Faculty of Palacky Univ. Olomouc 155.

Kuhn, M., Weston, S., Wing, J., Forester, J., 2016. The Contrast Package.
Lavoie, R.A., Jardine, T.D., Chumchal, M.M., Kidd, K.A., Campbell, L.M., 2013. Biomagnification of mercury in aquatic food webs: a worldwide meta-analysis. Environ. Sci. Technol. 47, 13385-13394.

Lefterova, M.I., Lazar, M.A., 2009. New developments in adipogenesis. Trends Endocrinol. Metabol. 20, 107-114.

Li, Y.-h., Liu, L., 2014. Apolipoprotein E synthesized by adipocyte and apolipoprotein E carried on lipoproteins modulate adipocyte triglyceride content. Lipids Health Dis. $13,136$.

Liu, Q., Basu, N., Goetz, G., Jiang, N., Hutz, R.J., Tonellato, P.J., Carvan, M.J., 2013. Differential gene expression associated with dietary methylmercury (MeHg) exposure in rainbow trout (Oncorhynchus mykiss) and zebrafish (Danio rerio) Ecotoxicology 22, 740-751.

Livak, K.J., Schmittgen, T.D., 2001. Analysis of relative gene expression data using real-time quantitative PCR and the $2-\Delta \Delta C T$ method. Methods 25, 402-408.

Louis, C., Van den Daelen, C., Tinant, G., Bourez, S., Thomé, J.-P., Donnay, I., Larondelle, Y., Debier, C., 2014. Efficient in vitro adipocyte model of long-term lipolysis: a tool to study the behavior of lipophilic compounds. In: Vitro Cellular \& Developmental Biology-Animal, vol. 50, pp. 507-518.

Lutfi, E., Riera-Heredia, N., Córdoba, M., Porte, C., Gutiérrez, J., Capilla, E., Navarro, I., 2017. Tributyltin and triphenyltin exposure promotes in vitro adipogenic differentiation but alters the adipocyte phenotype in rainbow trout. Aquat. Toxicol. 188, 148-158.

Mahaffey, K.R., Clickner, R.P., Bodurow, C.C., 2004. Blood organic mercury and dietary mercury intake: national health and nutrition examination survey, 1999 and 2000. Environ. Health Perspect. 112, 562-570.

Mauderly, J.L., Samet, J.M., 2008. Is there evidence for synergy among air pollutants in causing health effects? Environ. Health Perspect. 117, 1-6.

Mazerik, J.N., Hagele, T., Sherwani, S., Ciapala, V., Butler, S., Kuppusamy, M.L. Hunter, M., Kuppusamy, P., Marsh, C.B., Parinandi, N.L., 2007. Phospholipase A2 activation regulates cytotoxicity of methylmercury in vascular endothelial cells. Int. J. Toxicol. 26, 553-569.

McElwee, M.K., Ho, L.A., Chou, J.W., Smith, M.V., Freedman, J.H., 2013. Comparative toxicogenomic responses of mercuric and methyl-mercury. BMC Genom. 14 698.

Meacham, C., Freudenrich, T., Anderson, W., Sui, L., Lyons-Darden, T., Barone Jr., S., Gilbert, M., Mundy, W., Shafer, T., 2005. Accumulation of methylmercury or polychlorinated biphenyls in in vitro models of rat neuronal tissue. Toxicol. Appl. Pharmacol. 205, 177-187.

Mitchell, F.E., Roy, L.A., Taylor, P.M., 2010. Iodothyronine interactions with the system L1 amino acid exchanger in 3T3-L1 adipocytes. J. Thyroid Res. 2010.

Newbold, R.R., Padilla-Banks, E., Jefferson, W.N., 2009. Environmental estrogens and obesity. Mol. Cell. Endocrinol. 304, 84-89.

Nogara, P.A., Oliveira, C.S., Schmitz, G.L., Piquini, P.C., Farina, M., Aschner, M., Rocha, J.B., 2019. Methylmercury's chemistry: from the environment to the mammalian brain. Biochim. Biophys. Acta Gen. Subj.

Olsvik, P.A., Amlund, H., Torstensen, B.E., 2011. Dietary lipids modulate methylmercury toxicity in Atlantic salmon. Food Chem. Toxicol. 49, 3258-3271.

Pinheiro, J., Bates, D., DebRoy, S., Sarkar, D., 2018. R Core Team (2018). nlme: linear and nonlinear mixed effects models. R Package Version 3, 1-137.

Rabenstein, D.L., Fairhurst, M.T., 1975. Nuclear magnetic resonance studies of the solution chemistry of metal complexes. XI. Binding of methylmercury by sulfhydryl-containing amino acids and by glutathione. J. Am. Chem. Soc. 97, 2086-2092.

Ríos, J.M., Lana, N.B., Berton, P., Ciocco, N.F., Altamirano, J.C., 2015. Use of wild trout for PBDE assessment in freshwater environments: review and summary of critical factors. Emerging Contaminants 1, 54-63.

Ritchie, J.W., Baird, F.E., Christie, G.R., Stewart, A., Low, S.Y., Hundal, H.S., Taylor, P.M. 2001. Mechanisms of glutamine transport in rat adipocytes and acute regulation by cell swelling. Cell. Physiol. Biochem. 11, 259-270.

Rizzetti, D.A., Corrales, P., Piagette, J.T., Uranga-Ocio, J.A., Medina-Gomez, G., Peçanha, F.M., Vassallo, D.V., Miguel, M., Wiggers, G.A., 2019. Chronic mercury at low doses impairs white adipose tissue plasticity. Toxicology 418, 41-50.

Rosen, E.D., Hsu, C.-H., Wang, X., Sakai, S., Freeman, M.W., Gonzalez, F.J., Spiegelman, B.M., 2002. C/EBP $\alpha$ induces adipogenesis through PPAR $\gamma$ : a unified pathway. Genes Dev. 16, 22-26.

Rosen, E.D., Walkey, C.J., Puigserver, P., Spiegelman, B.M., 2000. Transcriptional regulation of adipogenesis. Genes Dev. 14, 1293-1307.

Salmerón, C., Acerete, L., Gutiérrez, J., Navarro, I., Capilla, E., 2013. Characterization and endocrine regulation of proliferation and differentiation of primary cultured preadipocytes from gilthead sea bream (Sparus aurata). Domest. Anim. Endocrinol. 45, 1-10.

Schirmer, K., 2006. Proposal to improve vertebrate cell cultures to establish them as substitutes for the regulatory testing of chemicals and effluents using fish. Toxicology 224, 163-183.

Schneider, A.-C., Beguin, P., Bourez, S., Perfield II, J.W., Mignolet, E., Debier, C., Schneider, Y.-J., Larondelle, Y., 2012. Conversion of t11t13 CLA into c9t11 CLA in Caco-2 cells and inhibition by sterculic oil. PLoS One 7, e32824.

Schwindt, A.R., Fournie, J.W., Landers, D.H., Schreck, C.B., Kent, M.L., 2008. Mercury concentrations in salmonids from western US national parks and relationships with age and macrophage aggregates. Environ. Sci. Technol. 42, 1365-1370.

Shanker, G., Hampson, R.E., Aschner, M., 2004. Methylmercury stimulates arachidonic acid release and cytosolic phospholipase A2 expression in primary neuronal cultures. Neurotoxicology 25, 399-406.

Shanker, G., Mutkus, L.A., Walker, S.J., Aschner, M., 2002. Methylmercury enhances arachidonic acid release and cytosolic phospholipase A2 expression in primary 
cultures of neonatal astrocytes. Mol. Brain Res. 106, 1-11.

Sherwani, S.I., Pabon, S., Patel, R.B., Sayyid, M.M., Hagele, T., Kotha, S.R. Magalang, U.J., Maddipati, K.R., Parinandi, N.L., 2013. Eicosanoid signaling and vascular dysfunction: methylmercury-induced phospholipase D activation in vascular endothelial cells. Cell Biochem. Biophys. 67, 317-329.

Sztalryd, C., Kimmel, A.R., 2014. Perilipins: lipid droplet coat proteins adapted for tissue-specific energy storage and utilization, and lipid cytoprotection. Biochimie 96, 96-101.

Telahigue, K., Rabeh, I., Hajji, T., Trabelsi, W., Bejaoui, S., Chouba, L., Soudani, N. 2019. Effects of acute mercury exposure on fatty acid composition and oxidative stress biomarkers in Holothuria forskali body wall. Ecotoxicol. Environ. Saf. 169, 516-522.

Todorčević, M., Škugor, S., Ruyter, B., 2010. Alterations in oxidative stress status modulate terminal differentiation in Atlantic salmon adipocytes cultivated in media rich in $\mathrm{n}-3$ fatty acids. Comp. Biochem. Physiol. B Biochem. Mol. Biol. 156, 309-318.

Ung, C.Y., Lam, S.H., Hlaing, M.M., Winata, C.L., Korzh, S., Mathavan, S., Gong, Z. 2010. Mercury-induced hepatotoxicity in zebrafish: in vivo mechanistic insights from transcriptome analysis, phenotype anchoring and targeted gene expression validation. BMC Genom. 11, 212

van den Dungen, M.W., Murk, A.J., Kok, D.E., Steegenga, W.T., 2017. Persistent organic pollutants alter DNA methylation during human adipocyte differentiation. Toxicol. Vitro 40, 79-87.
Vegusdal, A., Sundvold, H., Gjøen, T., Ruyter, B., 2003. An in vitro method for studying the proliferation and differentiation of Atlantic salmon preadipocytes. Lipids 38, 289-296.

Verrey, F., 2003. System L: heteromeric exchangers of large, neutral amino acids involved in directional transport. Pflügers Archiv 445, 529-533.

Verri, T., Terova, G., Romano, A., Barca, A., Pisani, P., Storelli, C., Saroglia, M., 2012. The SoLute Carrier (SLC) family series in teleost fish. Functional Genomics in Aquaculture 219-320.

Vertigan, T., Dunlap, K., Reynolds, A., Duffy, L., 2015. Effects of Methylmercury Exposure on 3T3-L1 Adipocytes. University of Alaska Fairbanks.

Wagemann, R., Trebacz, E., Boila, G., Lockhart, W., 1998. Methylmercury and total mercury in tissues of arctic marine mammals. Sci. Total Environ. 218, 19-31.

WHO, W.H.O., 2018. Obesity and Overweight. Fact Sheets. World Health Organ, Geneva, Switzerland.

Wu, G., Fang, Y.-Z., Yang, S., Lupton, J.R., Turner, N.D., 2004. Glutathione metabolism and its implications for health. J. Nutr. 134, 489-492.

Yadetie, F., Bjørneklett, S., Garberg, H.K., Oveland, E., Berven, F., Goksøyr, A., Karlsen, O.A., 2016. Quantitative analyses of the hepatic proteome of methylmercury-exposed Atlantic cod (Gadus morhua) suggest oxidative stressmediated effects on cellular energy metabolism. BMC Genom. 17, 554.

Ynalvez, R., Gutierrez, J., Gonzalez-Cantu, H., 2016. Mini-review: toxicity of mercury as a consequence of enzyme alteration. Biometals 29, 781-788. 